\title{
ON A CLASS OF NEW INEQUALITIES
}

\author{
BY
}

\author{
DANIEL T. SHUM
}

ABSTRACT. Inequalities of considerable interest are associated with the names of Beesack, Benson, Boyd, Calvert, Das, Hardy, Hua, Opial, Wong and Yang.

In this note an elementary method used in a recent paper by Benson will be further investigated. The resultant new class of inequalities will bring a great number of inequalities-such as inequalities of Hardy's and those of Opial's-under one roof, so to speak.

\section{CHAPTER 1. INTRODUCTION}

Inequalities of considerable interest are associated with the names of Beesack, Benson, Boyd, Calvert, Das, Hardy, Hua, Opial, Wong and Yang. The present note is a continuation of the author's previous papers [27], [28] in that Benson's method will be further investigated here. Actually, we are going to combine Benson's method with that of Beesack. In a number of papers ([2] - [5]), Beesack dealt with certain integral inequalities involving a function and its derivative. One of those results [5, Theorem 3.1.1] is a generalization of Hardy's inequality, and may be stated as follows:

Suppose that the differential equation

$$
(d / d x)\left[r\left(y^{\prime}\right)^{p-1}\right]+s y^{p-1}=0 \quad(p>1)
$$

has a solution $y(x)$ such that $y(x)>0, y^{\prime}(x)>0$ on $(a, b)$, and that

$$
y^{\prime} / y=O\left[(x-a)^{-1}\right] \text { as } x \rightarrow a+.
$$

If $r(x)=O\left[(x-a)^{p-1}\right] \quad\left(\right.$ or $\left.[r(x)]^{q / p} \int_{a}^{x} r^{-q / p} d t=O(x-a)\right)$, and $\int_{a}^{b} r\left(u^{\prime}\right)^{p} d x<$ $\infty$, then for every integral $u(x)=\int_{a}^{x} u^{\prime} d t$ (with $u^{\prime}$ nonnegative), we have

$$
\int_{a}^{b} s u^{p} d x \leqslant \int_{a}^{b} r\left(u^{\prime}\right)^{p} d x
$$

Here, $s(x), r(x), r^{\prime}(x)$ are assumed continuous on an interval $a<x<b$, and $r(x)>0, s(x) \geqslant 0$ on this interval, while $a$ or $b$ or both, may be infinite, and $p, q$ are conjugate exponents for Hölder's inequality.

Received by the editors August 10, 1973 and, in revised form, January 12, 1974. AMS (MOS) subject classifications (1970). Primary 26A84; Secondary 34B15.

Key words and phrases. Integral inequalities, differential boundary value problems, Riccati-like equation, Benson's method, Hardy type inequalities, Opial type inequalities. 
Basically, the method used in [2] - [5] depends on a relationship between the solution of certain differential boundary value problems and corresponding related integral inequalities. For (1.2), for example, the related boundary value conditions are

$$
\begin{gathered}
\int_{a}^{b} r\left(y^{\prime}\right)^{p} d x<\infty \text { with } y(x)=\int_{a}^{x} y^{\prime} d t, \\
\lim _{x \rightarrow b-} r(x) y(x)\left[y^{\prime}(x)\right]^{p-1}=0 .
\end{gathered}
$$

If the solution $y$ of (1.1) satisfies (A), (B), then equality will hold in (1.2) for the very solution of (1.1), and consequently the inequality (1.2) is sharp (that is, the unit constant on the right side is the best possible).

Moreover, if $\int_{a}^{b} r\left(y^{\prime}\right)^{p} d x=\infty$, then (1.2) is still sharp if either

$$
\varliminf_{x \rightarrow a^{+}} r(x) y(x)\left[y^{\prime}(x)\right]^{p-1}<\infty \text { or } \varliminf_{x \rightarrow b_{-}} r(x) y(x)\left[y^{\prime}(x)\right]^{p-1}<\infty .
$$

(Compare these boundary conditions with those in (A) and (B) above.)

In this way the author in [5] treated, thoroughly and elegantly, the extensions of Hardy's inequality for integrals [16, pp. 240-243]: if $p>1, u(x)=$ $\int_{0}^{x} u^{\prime} d t$ with $u^{\prime}(x) \geqslant 0$, then

$$
\int_{0}^{\infty} x^{-p} u^{p} d x<\left(\frac{p}{p-1}\right)^{p} \int_{0}^{\infty}\left(u^{\prime}\right)^{p} \cdot d x
$$

unless $u^{\prime} \equiv 0$. The constant is best possible.

Hardy's inequality is the special case of the quoted theorem having $a=0$, $b=\infty, y(x)=x^{(p-1) / p}$. (We obtain both Hardy's inequality and the above generalization of it as special cases of more general theorems in Chapter 2.)

Now returning to Benson's method we find that all results in [10] are based on the elementary algebraic inequality

$$
\begin{aligned}
x^{2 n}-2 n x+2 n-1 & >0 \text { for all real } x \neq 1, \\
& =0 \text { for } x=1,
\end{aligned}
$$

where $n$ is any positive integer.(1) (See also [22, pp. 126-129, 226].) Explicitly, Benson's method consists of the following steps (see also [22, p. 126]):

(1) Start from (1.3) to show that an expression which contains a function together with its derivative is nonnegative.

(2) To both sides of the inequality add something which is an exact derivative.

(1) See the comments in Zbl. Math. 146 (1968), 75-76, by P. R. Beesack, related to the paper of D. C. Benson. 
(3) Integrate both sides of the inequality to obtain the desired inequality. Here, we unify the above-mentioned two methods (i.e. Beesack's method in [5] and Benson's method in [10]) to produce integral inequalities which were neither handled in [5], nor in [10]. We bring all these inequalities, including those in [5] and in [10], under one roof, so to speak.

The reason why these two methods, which in appearance have no connection with each other, can be combined together, lies in the fact that they both use certain elementary algebraic inequalities as their starting point. This became more apparent when Benson's method was further developed in [27] and [28].

Although the following inequalities (1.4), (1.5), and Lemma 1.1 were already used in [28], for completeness we shall quote these inequalities and the lemma (but without proof) here.

Now, instead of (1.3) the elementary algebraic inequalities which we shall use in the sequel are as follows ([1] or [16, Theorem 41]):

$$
\begin{gathered}
s^{p}+(p-1) t^{p}-p s t^{p-1} \geqslant 0 \quad(p>1 \text { or } p<0) ; \\
s^{p}+(p-1) t^{p}-p s t^{p-1} \leqslant 0 \quad(0<p<1) .
\end{gathered}
$$

Here, $s$ and $t$ are nonnegative (positive if $p<0$ ), and in both cases strict inequality holds unless $s=t$. (We also note that when $p=0$ or $p=1$, the left sides of both (1.4) and (1.5) become identically zero for all $s$ and $t$.) For $p=2 n$ with $n$ a positive integer and $t=1,(1.4)$ is valid for any real $s$, since in this case (1.4) coincides with (1.3).

The associated integral inequalities are stated in the following lemma. The special case $p=2 n$ of (1.6) below gives the basic integral inequality used by Benson in [10].

LEMMA 1.1. Let $v(x)$ be absolutely continuous on $[\alpha, \beta]$ with $v^{\prime}(x) \geqslant$ 0 a.e. Also, suppose that $Q(x)$ is nonnegative a.e. and measurable on $[\alpha, \beta]$, and $G(v, x)$ is continuously differentiable for $x \in[\alpha, \beta]$, and $v$ in the range of the function $v(x)$, with $G_{v}(v, x) \geqslant 0$.(2) Then, if the integrals exist,

$$
\int_{\alpha}^{\beta}\left[Q v^{\prime p}+(p-1)\left(G_{v}\right)^{p /(p-1)} Q^{-1 /(p-1)}+p G_{x}\right] d x
$$

$$
\begin{aligned}
& \geqslant p[G(v(\beta), \beta)-G(v(\alpha), \alpha)](p>1 \text { or } p<0), \\
& \int_{\alpha}^{\beta}\left[Q v^{\prime p}+(p-1)\right.\left.\left(G_{v}\right)^{p /(p-1)} Q^{-1 /(p-1)}+p G_{x}\right] d x \\
& \leqslant p[G(v(\beta), \beta)-G(v(\alpha), \alpha)] \quad(0<p<1),
\end{aligned}
$$

(2) We note that in case $p=2 n$ with $n$ a positive integer, the restrictions $v^{\prime}(x)>$ 0 a.e., and $G_{v}(v, x)>0$ may be removed. 
where $G_{v}=(\partial / \partial v) G(v, x), G_{x}=(\partial / \partial x) G(v, x)$. Equality in both (1.6) and (1.7) holds if and only if the differential equation

$$
v^{\prime}=\left(G_{v} / Q\right)^{1 /(p-1)}
$$

is satisfied almost everywhere.

The details of our results will be presented in the next three chapters. In Chapter 2 we give further extensions of Opial's inequality. These extensions have the form

$$
\int_{a}^{b} S|u|^{p}\left|u^{\prime}\right|^{q} d x \leqslant \frac{q}{p+q} \int_{a}^{b} R\left|u^{\prime}\right|^{p+q} d x
$$

where $p, q$ are constants such that either $p \geqslant 0, q \geqslant 1$, or $p<0, q<0$, and the functions $R, S$ are related to the coefficients $r, s$ in a boundary value problem involving the differential equation

$$
(d / d x)\left\{r(x)\left[y^{\prime}(x)\right]^{p / q}\right\}=s^{\prime}(x)[y(x)]^{p / q} .
$$

The admissible functions $u$ for (1.9) are integrals on $[a, b)$ with $u(a)=0$, and $\int_{a}^{b} R\left|u^{\prime}\right|^{p+q} d x<\infty$.

The inequality (1.9) and the differential equation (1.10) were discussed by Boyd and Wong in [11], but with $q=1 \quad(p>0)$. Moreover, the equation (1.10) with $q=1$ was considered as long as ten years ago when Eeesack used it, for the first time, to obtain Hardy type inequalities. Also, inequalities of the form (1.9) were considered in a later paper [9] by Beesack and Das, but without relating them to a corresponding boundary value problem. We thus generalize the results of Boyd and Wong as well as unify some of the main results obtained in 1968 by Beesack and Das [9].

Although our original purpose was to get the generalization of Opial's original inequality, the essential part in this chapter is $\S 2.3$, in which we discuss the sharpness concerning the inequalities of the form (1.9) when the solution $y$ of (1.10) is not admissible, that is, $\left.\left.\int_{a}^{b} R\right|^{\prime}\right|^{p+q} d x=\infty$. In this section, as well as in the last two chapters, Beesack's method of showing that the constants are best possible is used. In $\$ 2.4$, a number of examples are given. One of them is as follows:

$$
\int_{a}^{b}(x-a)^{-p}|u|^{p}\left|u^{\prime}\right| d x \leqslant\left(\frac{p}{p+1}\right)^{-p} \int_{a}^{b}\left|u^{\prime}\right|^{p+1} d x \quad(p>0),
$$

valid for any $u$ such that $u(x)=\int_{a}^{x} u^{\prime} d t$ for $x \in(a, b)$ and $\int_{a}^{b}\left|u^{\prime}\right|^{p+1} d x<$ $\infty$, where $-\infty<a<b \leqslant \infty$. Equality holds in (1.11) if and only if $u \equiv 0$.

The constant is best possible.

In the last two chapters we use another differential boundary value problem 
to produce another new class of inequalities having the form

$$
\begin{gathered}
\left(\int_{a}^{b} W|u|^{p}\left|u^{\prime}\right|^{q} d x\right)^{k /(p+q)}+\left(\frac{q}{p+q}\right)^{k /(p+q)} C^{-1} \int_{a}^{b} S|u|^{k} d x \\
\leqslant\left(\frac{q}{p+q}\right)^{k /(p+q)} C^{-1} \int_{a}^{b} R\left|u^{\prime}\right|^{k} d x .
\end{gathered}
$$

Here either $p>0, k>q \geqslant 1$ or $p<0, k<q<0$ or $p>0, k<0<$ $q \leqslant 1 ; R, S$ are related to the differential coefficients appearing in the boundary value problem, and $W$ involves an arbitrary nonnegative function, while $u$ is any one of a suitable class of functions. Some inequalities related to (1.12) are also obtained. A reversed inequality of (1.12), that is, the inequality (1.12) with the direction of the inequality sign reversed, is also studied. The inequalities of Hardy [1, Theorem 330] and those of Opial's type are connected by (1.12), which contains, as special cases, both quoted inequalities. Opial's original inequality is obtained from (1.12) by setting $p=q=1, k=2, W=R \equiv 1, S \equiv 0$, and $a=0<b<\infty$. Hardy's inequality turns out to be a very special case of (1.12) with some modifications. Formally, Hardy's inequality is the special case of (1.12) having $\left.W \equiv 0, a=0, b=\infty, R=x^{k-r}, S=(|r-1|) / k\right)^{k} x^{-r}$, where $k>1, r \neq 1$, although the actual derivation of Hardy's inequality in $\S 3.4$ does not proceed in this way. Finally, we note that in 1969, Boyd [12] had previously considered inequalities of the form (1.12) but with the second term replaced by zero.

\section{Chapter 2. Further Extensions and}

\section{GeNERALIZATIONS OF OPIAL'S INEQUALITY}

2.1. Introduction. We shall now give some further results related to Opial's integral inequality. These results differ from those in [28] in that the latter provided a general and sharpened form of Opial's inequality, whereas the following theorems extend and generalize Opial's inequality in other directions. Moreover, in Chapter 3 we shall consider other extensions of Opial's inequality in a quite different manner. It will be found that some results in this chapter are covered by Chapter 3; nevertheless it is worth doing these separately because the nature of those proofs is quite different. The proof in the present chapter will be carried out by using Benson's method together with some Riccati-like equations whose simple form was used by Boyd and Wong [11]. It may not be without interest to note that in [11] the authors mentioned that their method was in turn suggested by a paper of P. R. Beesack [5].

In 1967 Boyd and Wong [11] proved the following type of extension of the inequality of Z. Opial [24]: 


$$
\int_{0}^{b} s|u|^{p}\left|u^{\prime}\right| d x \leqslant C \int_{0}^{b} r\left|u^{\prime}\right|^{p+1} d x
$$

where $b<\infty$, and the weighting functions $r$ and $s$ are nonnegative in $C^{1}[0, b]$, satisfying some Riccati-like equation. For $r(x)=s(x) \equiv 1$, and $p=$ 1, (2.2.1) reduces to Opial's inequality. (See also Olech [23], Beesack [6], Levinson [19], Mallows [20], and Pederson [25] for successively simpler proofs of Opial's inequality; as well as Redheffer [26], Beesack and Das [9], Boyd [12], [13] for other generalizations of this inequality.)

The purpose of this chapter is to establish inequalities of the following type:

$$
\int_{a}^{b} S|u|^{p}\left|u^{\prime}\right|^{q} d x \leqslant \frac{q}{p+q} \int_{a}^{b} R\left|u^{\prime}\right|^{p+q} d x,
$$

valid for all $p, q$ such that either $p \geqslant 0, q \geqslant 1$ or $p<0, q<0$.

The inequality (2.1.1) is a special case of (2.1.2) with $q=1, p \geqslant 0, a=$ 0 and $b<\infty$. (See also \$2.5.)

2.2. Main results. In this section we shall only prove Theorem 2.1 below. In order to prove this theorem we suppose that the following boundary value problem
(a) $\frac{d}{d x}\left\{r(x)\left[y^{\prime}(x)\right]^{p / q}\right\}=s^{\prime}(x)[y(x)]^{p / q}$,
(b) $\lim _{x \rightarrow a+} y\left\{r\left(y^{\prime}\right)^{p / q}-s y^{p / q}\right\}=0$,

$$
\begin{aligned}
& \text { (c) } \lim _{x \rightarrow b-} y\left\{r\left(y^{\prime}\right)^{p / q}-s y^{p / q}\right\}=0, \\
& \text { (d) } r(x)\left(y^{\prime} / y\right)^{p / a}\left(\int_{a}^{x} r^{-q / p} d t\right)^{p / q}=O(1) \text { as } x \rightarrow a+, \\
& \text { (e) } \int_{a}^{b} r\left(y^{\prime}\right)^{(p+q) / q} d x<\infty,
\end{aligned}
$$

has a solution $y=\int_{a}^{x} y^{\prime} d t$ with $y^{\prime}>0$ in $(a, b)(a$ or $b$ or both may be infinite), where $r$ is positive in $C(a, b)$ such that $\int_{a}^{b} r^{-q / p} d x<\infty$, and $s$ is nonnegative in $C^{\prime}(a, b)$. Here $r$ and $s$ are related to $R, S$ by (2.2.3) below, and if $p=0$, we assume that $r=s$. After setting $h=\left(y^{\prime} / y\right)^{p / q}$, we see that $h$ satisfies the Riccati-like equation

$$
(r h)^{\prime}+(p / q) r h^{(p+q) / p}=s^{\prime}(x) \text { for } x \in(a, b) \text { and } p \neq 0 .
$$

(Equations (2.2.1a) and (2.2.2) with $q=1, p>0$ were used by Boyd and Wong in [11].)

THEOREM 2.1. Let $p \geqslant 0, q \geqslant 1$, or $p<0, q<0$, and suppose that $r$, $s$ 
satisfy the conditions listed below (2.2.1). Let $y=\int_{a}^{x} y^{\prime} d t$, having $y^{\prime}>0$, be a solution of the boundary value problem (2.2.1). Let $w$ be a positive, measurable function on $(a, b)$ such that $\int_{a}^{x} w^{-1} d t<\infty$ for $x \in(a, b)$, and define the functions $R, S$ on $(a, b)$ by

$$
\begin{aligned}
& R(x)=r(x)[w(x)]^{(p+q)(q-1) / q} \\
& S(x)=s(x)[w(x)]^{q-1}\left(\int_{a}^{x} w^{-1} d t\right)^{p(1-q) / q} .
\end{aligned}
$$

- Finally, let $u$ be any function which is locally absolutely continuous on $(a, b)$ such that $u(x)=\int_{a}^{x} u^{\prime} d t$, and for which $\int_{a}^{b} R\left|u^{\prime}\right|^{p+q} d x<\infty$. Then

$$
\int_{a}^{b} S(x)|u|^{p}\left|u^{\prime}\right|^{q} d x \leqslant \frac{q}{p+q} \int_{a}^{b} R(x)\left|u^{\prime}\right|^{p+q} d x,
$$

valid for all $p, q$ such that $p \geqslant 0, q \geqslant 1$. If, in addition, $u^{\prime}$ does not change sign on $(a, b)$, then (2.2.4) is still valid for all $p, q$ such that $p<0, q<0$.

Equality holds in (2.2.4) for $p \neq 0$ if and only if

$$
\begin{array}{ll}
u=k_{1} y & \left(k_{1} \text { a constant }\right), \\
w=k_{2}\left(y^{\prime}\right)^{-1} & \left(k_{2}>0, a \text { constant }\right) \quad(q \neq 1),
\end{array}
$$

or $u=k_{1} y$ if $q=1$. If $p=0,(2.2 .4)$ reduces to an identity in $u$.

In order to prove this theorem, we state the following lemma, the proof of which can be accomplished by using Holder's inequality.

Lemma 2.1. Let $p>0, q>0$ or $p<0, q<0$ and suppose that $\int_{a}^{b} R \mid u^{\prime} p+q d x<\infty$, and $r(x) h(x)\left(\int_{a}^{x} r^{-q / p} d t\right)^{p / q}=O(1)$ as $x \rightarrow a+$, as stated in the above hypotheses of Theorem 2.1. Then $\int_{a}^{x} w^{q-1}\left|u^{\prime}\right|^{q} d x<\infty$ for $x \tilde{\epsilon}(a, b)$, and

$$
\lim _{x \rightarrow a+}\left(\int_{a}^{x} w^{q-1}\left|u^{\prime}\right|^{q} d t\right)^{(p+q) / q} r(x) h(x)=0 .
$$

If, in addition, we assume that $\int_{a}^{b} r^{-q / p} d x<\infty$, then $\int_{a}^{b} w^{q-1}\left|u^{\prime}\right|^{q} d x<\infty$.

Proof OF TheOREM 2.1. First we note that, when $p=0,(2.2 .4)$ becomes an identity in $u$, so we may assume that $p \neq 0$. Now, from (1.6) on replacing $p$ by $(p+q) / q$, we have, for $(p+q) / q>1$,

$$
\int_{\alpha}^{\beta}\left\{Q\left(v^{\prime}\right)^{(p+q) / q}+(p / q)\left(G_{v}\right)^{(p+q) / p} Q^{-q / p}+[(p+q) / q] G_{x}\right\} d x
$$

$$
\geqslant\left.[(p+q) / q] G(v, x)\right|_{\alpha} ^{\beta} \text {. }
$$

By (1.8), on replacing $p$ by $(p+q) / q$, equality holds in (2.2.7) if and only if 


$$
v^{\prime}=\left(G_{v} / Q\right)^{q / p} \text {, a.e. }
$$

Now, let $z(x)=\int_{a}^{x} w^{q-1}\left|u^{\prime}\right|^{q} d t$ which is well defined by Lemma 2.1 . In (2.2.7), set

$$
v=\int_{a}^{x} w^{q-1}\left|u^{\prime}\right|^{q} z^{p / q} d t=[q /(p+q)] z^{(p+q) / q}
$$

(since $(p+q) / q>0), Q=r z^{-p(p+q) / q^{2}}, G=v(r h)$, and on using (2.2.2) we have, for $a<\alpha<\beta<b$,

$$
\begin{aligned}
\int_{\alpha}^{\beta} R\left|u^{\prime}\right|^{p+q} d x+\int_{\alpha}^{\beta} s^{\prime} z^{(p+q) / q} d x \\
\quad \geqslant[z(\beta)]^{(p+q) / q} r(\beta) h(\beta)-[z(\alpha)]^{(p+q) / q} r(\alpha) h(\alpha) .
\end{aligned}
$$

By integrating by parts, (2.2.9) reduces to

$$
\begin{aligned}
& \int_{\alpha}^{\beta} R\left|u^{\prime}\right|^{p+q} d x+[z(\alpha)]^{(p+q) / q} r(\alpha) h(\alpha) \\
& \geqslant {[z(\beta)]^{(p+q) / q} r(\beta) h(\beta)+[z(\alpha)]^{(p+q) / q} s(\alpha) } \\
&-[z(\beta)]^{(p+q) / q} s(\beta)+[(p+q) / q] \int_{\alpha}^{\beta} s w^{q-1} z^{p / q}\left|u^{\prime}\right|^{q} d x .
\end{aligned}
$$

Now by letting $\alpha \rightarrow a+$, on using (2.2.6), and the fact that $s(x) \geqslant 0$ noted following (2.2.1), we have

$$
\begin{aligned}
\int_{a}^{\beta} R\left|u^{\prime}\right|^{p+q} d x \geqslant & {[z(\beta)]^{(p+q) / q}[r(\beta) h(\beta)-s(\beta)] } \\
& +\frac{p+q}{q} \int_{a}^{\beta} s w^{q-1} z^{p / q}\left|u^{\prime}\right|^{q} d x .
\end{aligned}
$$

On noting the definition of $h(x)$, and using the last assertion in Lemma 2.1 and the boundary condition (2.2.1c), together with the fact that $y^{\prime}(x)$ is positive and $y(x)$ is increasing on $(a, b)$, we finally get, on letting $\beta \rightarrow b$ in (2.2.11),

$$
\int_{a}^{b} s w^{q-1} z^{p / q}\left|u^{\prime}\right|^{q} d x \leqslant\left[\frac{q}{p+q}\right] \int_{a}^{b} R\left|u^{\prime}\right|^{p+q} d x .
$$

Now, using Hölder's inequality with indices $q$ and $q^{\prime}=q /(q-1)$, we have (in case $p>0, q>1$ ),

$$
\begin{aligned}
& |u|^{p}=\left|\int_{a}^{x} u^{\prime} d t\right|^{p} \leqslant\left(\int_{a}^{x}\left|u^{\prime}\right| d t\right)^{p}=\left(\int_{a}^{x} w^{1 / q^{\prime}}\left|u^{\prime}\right| w^{-1 / q^{\prime}} d t\right)^{p} \\
& \leqslant\left(\int_{a}^{x} w^{q-1}\left|u^{\prime}\right|^{q} d t\right)^{p / q}\left(\int_{a}^{x} w^{-1} d t\right)^{p(q-1) / q} .
\end{aligned}
$$

That is, 


$$
\left(\int_{a}^{x} w^{-1} d t\right)^{p(1-q) / q}|u|^{p} \leqslant z^{p / q} .
$$

On noting the definition of $S(x)$, the proof of (2.2.4) is completed by combining (2.2.12) and (2.2.14).

Equality holds in (2.2.13), and hence in (2.2.14), if and only if $u^{\prime}$ does not change sign, and $w^{q-1}\left|u^{\prime}\right|^{q}=k_{0} w^{-1} \quad\left(k_{0}>0\right.$, a constant $)$, or

$$
u^{\prime}=k w^{-1} \quad(k \text { a constant }) \text {. }
$$

If $u^{\prime}$ does not change sign on $(a, b)$, then the first inequality in (2.2.13) becomes an equality, and consequently (2.2.13), (2.2.14) and the equality condition (2.2.15) are still valid for all $p, q$ such that $p q>0$ and $p+q<0$. (We also note that when $q=1,(2.2 .14)$ is still true, and equality holds in this case if and only if $u^{\prime}$ does not change sign.)

Now, by using (2.2.8), equality holds in (2.2.10) if and only if $w^{q-1}\left|u^{\prime}\right|^{q} z^{p / q}=n^{q / p} z^{(p+q) / q}$, or $z^{\prime} / z=n^{q / p}=y^{\prime} / y$. That is, $z^{\prime}=c y^{\prime}$, for some positive constant $c$. Hence by using the definition of $z$, equality holds in (2.2.10) if and only if

$$
\left|u^{\prime}\right|=\left(c y^{\prime}\right)^{1 / q} w^{(1-q) / q} .
$$

This, together with (2.2.15), means that equality can hold in (2.2.4) only if, (for $q \neq 1$ ),

$$
\begin{array}{ll}
u=k_{1} y & \left(k_{1} \quad \text { a constant }\right) \\
w=k_{2}\left(y^{\prime}\right)^{-1} & \left(k_{2}>0 \quad \text { a constant }\right)
\end{array}
$$

With $u, w$ so defined by (2.2.17) it is easy to show that $w$ is well defined, that $u$ is admissible (that is, $u$ satisfies the hypotheses stated in the theorem), and that equality in (2.2.4) is attained. We only point out that in the course of these proofs we need $(2.2 .1 \mathrm{a}, \mathrm{b}, \mathrm{c}, \mathrm{e})$, together with a repeated use of integration by parts.

For $q=1$, equality can hold in (2.2.4) only if $u^{\prime}$ does not change sign (by a remark following (2.2.15)), and $\left|u^{\prime}\right|=c y^{\prime}$ by (2.2.16). Hence, equality can hold in (2.2.4) for $q=1$ only if $u=k_{1} y$ ( $k_{1}$ a constant). As pointed out in the above paragraph, we can again get equality in (2.2.4) in this case, proving the theorem.

REMARK 2.1. We note that the assumption (2.2.1e), which will be removed in the following section, together with the fact that $w=k_{2}\left(y^{\prime}\right)^{-1}$ is well defined, ensures the existence of a nontrivial admissible function, namely the very solution $y$ of (2.2.1).

REMARK 2.2. Although we only prove Theorem 2.1 here, we may establish 
another theorem with $u(b)=0$, by interchanging the roles of $a$ and $b$ in Theorem 2.1. Actually, in order to obtain the Opial's original inequality as a special case, one needs both Theorem 2.1 and the corresponding theorem with $u(b)=0$.

2.3. Sharpness of the inequalities when $y$ is not admissible. We are now going to deal with those cases where the inequality (2.2.4) is best possible, but equality is only attained in the trivial case $u \equiv 0$ (or never in case $p<0, q<$ 0 ). We first note that when $w=\left(y^{\prime}\right)^{-1},(2.2 .4)$ reduces to

$$
\begin{aligned}
\int_{a}^{b} s\left(y^{\prime}\right)^{1-q} y^{p(1-q) / q}|u|^{p}\left|u^{\prime}\right|^{q} d x & \\
& \leqslant \frac{q}{p+q} \int_{a}^{b} r\left(y^{\prime}\right)^{(p+q)(1-q) / q}\left|u^{\prime}\right|^{p+q} d x .
\end{aligned}
$$

In order to duscuss the sharpness of (2.3.1), we must now consider separately the two cases $p>0, q \geqslant 1$ and $p<0, q<0$, as the details differ in the two cases. The basic ideas in these subsections were adapted from [5].

2.3.1. The case $p>0, q \geqslant 1$. The hypotheses as stated in Theorem 2.1 are unchanged except that instead of (2.2.1) we now assume
(a) $\frac{d}{d x}\left\{r(x)\left[y^{\prime}(x)\right]^{p / q}\right\}=s^{\prime}(x)[y(x)]^{p / q}$,
(b) $y\left[r\left(y^{\prime}\right)^{p / q}-s y^{p / q}\right]=O(1)$ as $x \rightarrow a+$
(c) $y\left[r\left(y^{\prime}\right)^{p / q}-s y^{p / q}\right]=o(1)$ as $x \rightarrow b-$,
(d) $r(x)\left(y^{\prime} / y\right)^{p / q}\left(\int_{a}^{x} r^{-q / p} d t\right)^{p / q}=O(1)$ as $x \rightarrow a+$,
(e) $\int_{a}^{b} r\left(y^{\prime}\right)^{(p+q) / q} d x=\infty$,

has a positive solution $y(x)=\int_{a}^{x} y^{\prime} d t$ such that $y^{\prime}(x)>0$ on $(a, b)$. (We note that (2.3.2a, c, d) ensure the validity of the inequality (2.3.1) itself as proved in Theorem 2.1, that (2.3.2b) is weaker than (2.2.1b), and that (2.3.2e) states that there exists no nontrivial admissible function for which equality can be attained in (2.3.1). ) Under these assumptions (2.3.1) is sharp, that is, the constant $q /(p+q)$ is best possible, although equality holds in (2.3.1) if and only if $u \equiv 0$.

To prove this we first note that for any $\delta>0$, by selecting $a^{\prime}$ or $b^{\prime}$, or perhaps both, appropriately close to $a$ or $b$, we have 


$$
\left(\frac{q(p+1)}{p}-\frac{q}{p+q}\right) y\left(a^{\prime}\right)\left\{r\left(a^{\prime}\right)\left[y^{\prime}\left(a^{\prime}\right)\right]^{p / q}-s\left(a^{\prime}\right)\left[y\left(a^{\prime}\right)\right]^{p / q}\right\}
$$

$$
\begin{aligned}
& +\left(\frac{q}{p+q}-\frac{q(p+1) y\left(a^{\prime}\right)}{p y\left(b^{\prime}\right)}\right) y\left(b^{\prime}\right)\left\{r\left(b^{\prime}\right)\left[y^{\prime}\left(b^{\prime}\right)\right]^{p / q}-s\left(b^{\prime}\right)\left[y\left(b^{\prime}\right)\right]^{p / q}\right\} \\
& <\frac{\delta q}{p+q} \int_{a^{\prime}}^{b^{\prime}} r\left(y^{\prime}\right)^{(p+q) / q} d x .
\end{aligned}
$$

This follows from $(2.3 .2 \mathrm{~b}, \mathrm{c}, \mathrm{e})$ and the fact that $y(x)$, with $y(a)=0$, is positive and increasing on $(a, b)$.

Now we take

$$
\begin{aligned}
u^{\prime}(x) & =0, & & a \leqslant x \leqslant a^{\prime}, \\
& =y^{\prime}(x), & & a^{\prime}<x<b^{\prime}, \\
& =0, & & b^{\prime} \leqslant x \leqslant b .
\end{aligned}
$$

Then $u(x)=y(x)-y\left(a^{\prime}\right)$ for $a^{\prime}<x<b^{\prime}$, and for $p>0$,

$$
\begin{aligned}
{[u(x)]^{p+1} } & =[y(x)]^{p+1}\left\{1-\frac{y\left(a^{\prime}\right)}{y(x)}\right\}^{p+1} \\
& >[y(x)]^{p+1}\left\{1-(p+1) \frac{y\left(a^{\prime}\right)}{y(x)}\right\}, \quad a^{\prime}<x<b^{\prime} .
\end{aligned}
$$

This inequality is the special case of (1.4) with $p$ replaced by $p+1$, obtained by setting $s=1-y\left(a^{\prime}\right)[y(x)]^{-1}, t=1$.

Now from (2.3.2a) we have

$$
\int_{a^{\prime}}^{b^{\prime}} y\left[r\left(y^{\prime}\right)^{p / q}\right]^{\prime} d x=\int_{a^{\prime}}^{b^{\prime}} s^{\prime} y^{(p+q) / q} d x .
$$

Integrating by parts reduces (2.3.6) to

$$
\begin{aligned}
\int_{a^{\prime}}^{b^{\prime}} s y^{p / q} y^{\prime} d x= & \frac{q}{p+q} \int_{a^{\prime}}^{b^{\prime}} r\left(y^{\prime}\right)^{(p+q) / q} d x \\
& +\left.\frac{q y}{p+q}\left[s y^{p / q}-r\left(y^{\prime}\right)^{p / q}\right]\right|_{a^{\prime}} ^{b^{\prime}} .
\end{aligned}
$$

Using (2.3.5) and (2.3.7), on noting that $s y^{\prime} y^{p(1-q) / q} \geqslant 0$, and $y(x)>$ $u(x)>0$ on $\left(a^{\prime}, b^{\prime}\right)$, we have

$$
\begin{aligned}
& \int_{a^{\prime}}^{b^{\prime}} s y^{\prime} y^{p(1-q) / q} u^{p} d x>\int_{a^{\prime}}^{b^{\prime}} s y^{[p(1-q) / q]-1} y^{\prime} u^{p+1} d x \\
& >\int_{a^{\prime}}^{b^{\prime}} s y^{\prime} y^{p / q} d x-(p+1) y\left(a^{\prime}\right) \int_{a^{\prime}}^{b^{\prime}} s y^{\prime} y^{(p-q) / q} d x \\
& =\frac{q}{p+q} \int_{a^{\prime}}^{b^{\prime}} r\left(y^{\prime}\right)^{(p+q) / q} d x+\left.\frac{q y}{p+q}\left[s y^{p / q}-r\left(y^{\prime}\right)^{p / q}\right]\right|_{a^{\prime}} ^{b^{\prime}} \\
& -(p+1) y\left(a^{\prime}\right) \int_{a^{\prime}}^{b^{\prime}} s y^{\prime} y^{(p-q) / q} d x
\end{aligned}
$$


Again, from (2.3.2a) we have

$$
\int_{a^{\prime}}^{b^{\prime}} s y^{(p-q) / q} y^{\prime} d x=\left.(q / p)\left[s y^{p / a}-r\left(y^{\prime}\right)^{p / q}\right]\right|_{a^{\prime}} ^{b^{\prime}}
$$

This and (2.3.8), together with (2.3.3), imply that for any $\delta>0$ there exists an admissible function $u$ defined by (2.3.4) such that

$$
\int_{a}^{b} s\left(y^{\prime}\right)^{1-q} y^{p(1-q) / q} u^{p}\left(u^{\prime}\right)^{q} d x
$$

$$
>(1-\delta) \frac{q}{p+q} \int_{a}^{b} r\left(y^{\prime}\right)^{(p+q)(1-q) / q}\left(u^{\prime}\right)^{p+q} d x .
$$

This proves the sharpness concerning (2.3.1).

2.3.2. The case $p<0, q<0$. Since in this case equality in (2.3.1) never occurs in view of our requirement $u^{\prime} \neq 0$ in $(a, b)$, we must modify the procedure used in 2.3.1. The hypotheses are the same as those in 2.3.1 except that we need the following additional stronger assumption,

$$
\lim _{x \rightarrow a+}\left[r\left(y^{\prime}\right)^{p / q}-s y^{p / q}\right]<\infty .
$$

We note that the limit in (2.3.11) always exist, finite or infinite, and that (2.3.11), together with $y(a)=0$, implies (2.2.1b). To see this, we set $f=$ $r\left(y^{\prime}\right)^{p / q}-s y^{p / q} ;$ then

$$
f^{\prime}=\left[r\left(y^{\prime}\right)^{p / q}\right]^{\prime}-s^{\prime} y^{p / q}-(p / q) s y^{(p-q) / q} y^{\prime}<0,
$$

by using (2.3.2a). Thus $f$ is monotone decreasing on $(a, b)$ and hence the existence (finite or infinite) of the limit in (2.3.11) follows.

Now, we set

$$
\begin{aligned}
u^{\prime}(x) & =g(x), & & a \leqslant x \leqslant a^{\prime}, \\
& =y^{\prime}(x), & & a^{\prime}<x<b^{\prime}, \\
& =g(x), & & b^{\prime} \leqslant x \leqslant b .
\end{aligned}
$$

Here $a^{\prime}, b^{\prime}$ are to be assigned, and $g(x) \geqslant 0$ is any (fixed) admissible function. For $a^{\prime}<x<b^{\prime}$, we have

$$
u(x)=\int_{a}^{x} u^{\prime} d t=y(x)\left[1-\frac{y\left(a^{\prime}\right)}{y(x)}+\frac{1}{y(x)} \int_{a}^{a^{\prime}} g d t\right] .
$$

A special case of (1.4) obtained by setting $s=1-y\left(a^{\prime}\right) / y(x)+\int_{a}^{a^{\prime}} g d t / y(x)$, $t=1$, gives us, for $p<0$,

$$
[u(x)]^{p}=[y(x)]^{p}\left(1-\frac{y\left(a^{\prime}\right)}{y(x)}+\frac{\int_{a}^{a^{\prime}} g d t}{y(x)}\right)^{p}
$$

$$
\geqslant[y(x)]^{p}\left(1-p \frac{y\left(a^{\prime}\right)}{y(x)}+p \frac{\int_{a}^{a^{\prime}} g d t}{y(x)}\right) \text { for } a^{\prime}<x<b^{\prime} .
$$


Now, since $s y^{\prime} y^{p(1-q) / q} \geqslant 0$, from (2.3.7), (2.3.9) and (2.3.14) it follows that (after simplifying)

$$
\begin{gathered}
\int_{a}^{b} s\left(y^{\prime}\right)^{1-q} y^{p(1-q) / q} u^{p}\left(u^{\prime}\right)^{q} d x \geqslant \frac{q}{p+q} \int_{a}^{b} r\left(y^{\prime}\right)^{(p+q)(1-q) / q}\left(u^{\prime}\right)^{p+q} d x \\
+\frac{q}{p+q}\left\{\left.y\left[s y^{p / q}-r\left(y^{\prime}\right)^{p / q}\right]\right|_{a^{\prime}} ^{b^{\prime}}\right\}
\end{gathered}
$$

$$
\begin{aligned}
& -q\left[y\left(a^{\prime}\right)-\int_{a}^{a^{\prime}} g d t\right]\left\{\left.\left[s y^{p / q}-r\left(y^{\prime}\right)^{p / q}\right]\right|_{a^{\prime}} ^{b^{\prime}}\right\} \\
& -\frac{q}{p+q} \int_{a}^{a^{\prime}} r\left(y^{\prime}\right)^{(p+q)(1-q) / q} g^{p+q} d x \\
& -\frac{q}{p+q} \int_{b^{\prime}}^{b} r\left(y^{\prime}\right)^{(p+q)(1-q) / q} g^{p+q} d x .
\end{aligned}
$$

Hence,

$$
\begin{aligned}
\int_{a}^{b} s y^{p(1-q) / q}\left(y^{\prime}\right)^{1-q} u^{p}\left(u^{\prime}\right)^{q} d x \\
\\
\geqslant \frac{(1-\delta) q}{p+q} \int_{a}^{b} r\left(y^{\prime}\right)^{(p+q)(1-q) / q}\left(u^{\prime}\right)^{p+q} d x,
\end{aligned}
$$

provided

$$
\begin{aligned}
& \left.\frac{q}{p+q} y\left[r\left(y^{\prime}\right)^{p / q}-s y^{p / q}\right]\right|_{a^{\prime}} ^{b^{\prime}}-\left.q y\left(a^{\prime}\right)\left[r\left(y^{\prime}\right)^{p / q}-s y^{p / q}\right]\right|_{a^{\prime}} ^{b^{\prime}} \\
& +q \int_{a}^{a^{\prime}} g d t\left\{r\left(b^{\prime}\right)\left[y^{\prime}\left(b^{\prime}\right)\right]^{p / q}-s\left(b^{\prime}\right)\left[y\left(b^{\prime}\right)\right]^{p / q}\right\} \\
& -q \int_{a}^{a^{\prime}} g d t\left\{r\left(a^{\prime}\right)\left[y^{\prime}\left(a^{\prime}\right)\right]^{p / q}-s\left(a^{\prime}\right)\left[y\left(a^{\prime}\right)\right]^{p / q}\right\} \\
& +\frac{q(1-\delta)}{p+q}\left\{\int_{a}^{a^{\prime}} r\left(y^{\prime}\right)^{(p+q)(1-q) / q} g^{p+q} d x\right. \\
& \left.\quad+\int_{b^{\prime}}^{b} r\left(y^{\prime}\right)^{(p+q)(1-q) / q} g^{p+q} d x\right\} \\
& <\frac{\delta q}{p+q} \int_{a^{\prime}}^{b^{\prime}} r\left(y^{\prime}\right)^{(p+q) / q} d x .
\end{aligned}
$$

To establish the fact that the inequality (2.3.17) can be satisfied by choosing $a^{\prime}$, $b^{\prime}$ appropriately near $a, b$ respectively, we only point out that the fourth term tends to zero, using our new assumption (2.3.11) together with the fact that $\int_{a}^{a^{\prime}} g d t \rightarrow 0$ as $a^{\prime} \rightarrow a+$. Thus from (2.3.16) the sharpness of (2.3.1) follows in this case. 
2.4. Some examples. Although in this section we only consider the case $u(a)=0$, the corresponding results for $u(b)=0$ may also be obtained. We only consider the case $w=\left(y^{\prime}\right)^{-1}$, so the inequalities thus obtained will give the best constants.

Now, let $\alpha<1$, and $-\infty<a<b<\infty$. In Theorem 2.1 set $r(x)=$ $(x-a)^{\alpha p / q}, s(x) \equiv(1-\alpha)^{p / q}(b-a)^{p(\alpha-1) / q}, w(x)=(x-a)^{\alpha}$. Then (2.2.1) has a solution $y(x)=(1-\alpha)^{-1}(x-a)^{1-\alpha}$ such that $w=\left(y^{\prime}\right)^{-1}$. All hypotheses of Theorem 2.1 are satisfied. Thus, by (2.2.4) we obtain, after some rearrangement,

$$
\begin{aligned}
& \int_{a}^{b}(x-a)^{(q-1)[\alpha(p+q)-p] / q}|u|^{p}\left|u^{\prime}\right|^{q} d x \\
& \quad \leqslant \frac{q}{p+q} \frac{(b-a)^{p(1-\alpha) / q}}{(1-\alpha)^{p}} \int_{a}^{b}(x-a)^{\alpha(p+q-1)}\left|u^{\prime}\right|^{p+q} d x .
\end{aligned}
$$

This inequality is valid for any function $u$ which is absolutely continuous on $[a, b)$ with $u(a)=0$, and for all $p, q$ such that either $p \geqslant 0, q \geqslant 1$ or $p<0$, $q<0$, if $u^{\prime}$ does not change sign in the latter case. Equality holds in (2.4.1) if and only if $u=c(x-a)^{1-\alpha}$, for some constant $c$.

EXAMPLE 2.1. Taking $\alpha=0$ in (2.4.1) we obtain

$$
\int_{a}^{b}(x-a)^{p(1-q) / q}|u|^{p}\left|u^{\prime}\right|^{q} d x \leqslant \frac{q}{p+q}(b-a)^{p / q} \int_{a}^{b}\left|u^{\prime}\right|^{p+q} d x,
$$

for any function $u$ which is absolutely continuous on $[a, b)$ with $u(a)=0$, and for all $p, q$ such that either $p \geqslant 0, q \geqslant 1$ or $p<0, q<0$, if $u^{\prime}$ does not change sign in the latter case. Equality holds in (2.4.2) if and only if $u=$ $c(x-a)$. (We note that $(2.4 .2)$ is obtained in $[9,(19)]$, but for a slightly broader range of values of $p, q$, namely, instead of $p \geqslant 0, q \geqslant 1,[9,(19)]$ is also valid for $p q>0$ and $p+q \geqslant 1$.)

EXAMPLE 2.2. Taking $\alpha=p /(p+q)$ in (2.4.1), we have

$$
\begin{aligned}
\int_{a}^{b}|u|^{p}\left|u^{\prime}\right|^{q} d x \leqslant & \left(\frac{q}{p+q}\right)^{1-p}(b-a)^{p /(p+q)} \\
& \times \int_{a}^{b}(x-a)^{p(p+q-1) /(p+q)}\left|u^{\prime}\right|^{p+q} d x,
\end{aligned}
$$

for any absolutely continuous function $u$ on $[a, b)$ having $u(a)=0$, and for all $p, q$ such that either $p \geqslant 0, q \geqslant 1$ or $p<0, q<0$, if $u^{\prime}$ does not change sign in the latter case. Equality holds in (2.4.3) if and only if $u=c(x-a)^{q /(p+q)}$. (This , again, is obtained in $[9,(20)]$; the same remark as in the previous example applies here.)

In order to give the next example, we need the following remark.

REMARK 2.3. In (2.2.1) we assumed that $\int_{a}^{b} r^{-q / p} d x<\infty$. However, we 
may remove this assumption provided

$$
y(x)\left\{r(x)\left[y^{\prime}(x)\right]^{p / q}-s(x)[y(x)]^{p / q}\right\} \geqslant 0 \text { for } x \text { near } b .
$$

(We note that (2.4.4) actually implies that $y\left[r\left(y^{\prime}\right)^{p / q}-s y^{p / q}\right] \geqslant 0$ for all $x \in(a, b)$, since $g=r\left(y^{\prime}\right)^{p / q}-s y^{p / q}$ is decreasing on $(a, b)$.) To see this we note that the condition $\int_{a}^{b} r-q / p d x<\infty$ was only used in the last assertion of Lemma 2.1, which, again, was only used to get (2.2.12) from (2.2.11). However, (2.4.4) automatically reduces (2.2.11) to (2.2.12). In Theorem 2.1, the other assumptions are retained; in particular, (2.1.2c) is still needed in order to get the cases of equality as asserted.

In $\S 2.3$, however, (2.3.2c) may now be replaced by (2.4.4) together with the following weaker assumption:

$$
y\left[r\left(y^{\prime}\right)^{p / q}-s y^{p / q}\right]=O(1) \text { as } x \rightarrow b-.
$$

We note that (2.4.4) ensures the validity of the inequality (2.3.1) itself, whereas only (2.4.5) is needed in (2.3.3) to assure the sharpness of (2.3.1). It is (2.4.4), together with (2.4.5), that is practically useful as we shall see in the following interesting examples.

Now let $-\infty<a<b \leqslant \infty$. In (2.3.2) we take $r \equiv((p+q) / p)^{(p+q) / q}$, $s=(q / p)(x-a)^{-p / q}$. Then (2.3.2), with (2.3.2c) replaced by (2.4.4) and (2.4.5), has a solution $y(x)=(x-a)^{p /(p+q)}$. The hypotheses at the beginning of subsection 2.3.1 are satisfied. Hence, by (2.3.1) we have, after simplifying:

$$
\int_{a}^{b}(x-a)^{q-p-1}|u|^{p}\left|u^{\prime}\right|^{q} d x \leqslant\left(\frac{p}{p+q}\right)^{-p} \int_{a}^{b}(x-a)^{q-1}\left|u^{\prime}\right|^{p+q} d x
$$

valid for any $u$ such that $u(x)=\int_{a}^{x} u^{\prime} d t$ for $x \in(a, b)$ and $\int_{a}^{b}(x-a)^{q-1}\left|u^{\prime}\right|^{p+q} d x<\infty$, and for all $p, q$ such that $p \geqslant 0, q \geqslant 1$. Equality holds in (2.4.6) if and only if $u \equiv 0$. The constant $[p /(p+q)]^{-p}$ is the best possible.

REMARK 2.4. We note that if $u^{\prime}$ is of one sign on $(a, b)$, then the inequality (2.4.6) is still valid for all $p, q$, such that $p<0, q<0$, provided $b=\infty$ there. To see this we note that when $b=\infty$, the integral appearing in (2.3.2e) diverges at $b$, that is that $\int_{X}^{\infty} r\left(y^{\prime}\right)^{(p+q) / q} d x=\infty$ for any $X \in(a, \infty)$ in our example. Now we fix $a^{\prime}$, and let $b^{\prime}$ tend to infinity in (2.3.1); the sharpness in our case follows from (2.4.5) and the specialized condition (2.3.2e).

EXAMPLE 2.3. Taking $q=1$ in (2.4.6) we have, for $p \geqslant 0$, and $-\infty<$ $a<b \leqslant \infty$,

$$
\int_{a}^{b}(x-a)^{-p}|u|^{p}\left|u^{\prime}\right| d x \leqslant\left(\frac{p}{p+1}\right)^{-p} \int_{a}^{b}\left|u^{\prime}\right|^{p+1} d x,
$$

valid for any $u$ such that $u(x)=\int_{a}^{x} u^{\prime} d t$ for $x \in(a, b)$ and $\int_{a}^{b}\left|u^{\prime}\right|^{p+1} d x<$ 
$\infty$. Equality holds in (2.4.7) if and only if $u \equiv 0$. The constant $[p /(p+1)]^{-p}$ is the best possible.

It may be of interest to compare (2.4.7) with $a=0, b=\infty$ to the following Hardy's inequality for integrals:

$$
\int_{0}^{\infty} x^{-p} u^{p} d x<\left(\frac{p}{p-1}\right)^{p} \int_{0}^{\infty}\left(u^{\prime}\right)^{p} d x \quad(p>1),
$$

unless $u^{\prime} \equiv 0$. The constant is the best possible. Here $u(x)=\int_{0}^{x} u^{\prime} d t$ with $u^{\prime} \geqslant 0$. (See also [27].)

Although we shall not give examples here, we finally note that if $w \neq$ $\left(y^{\prime}\right)^{-1}$, then (2.2.4) is, in general, not sharp.

2.5. Comparison of results. We first wish to show that the results in this chapter are direct generalizations of those in [11]. In [11] the boundary value problem

$$
\begin{aligned}
& \text { (a) }(d / d x)\left\{r(x)\left[y^{\prime}(x)\right]^{p}\right\}=\lambda s^{\prime}(x)[y(x)]^{p} \\
& \text { (b) } r(b)\left[y^{\prime}(b)\right]^{p}=\lambda s(b)[y(b)]^{p}, \\
& \text { (c) } y(0)=0
\end{aligned}
$$

was considered. Here $b<\infty$, and $r$ and $s$ are nonnegative in $C^{1}[0, b]$. The authors in [11] assumed that (2.5.1) has a solution $y$ for which $y^{\prime}>0$ in $[0, b]$. Under these assumptions they obtained the inequality

$$
\begin{aligned}
& \int_{0}^{b} s(x)|u(x)|^{p}\left|u^{\prime}(x)\right| d x \\
& \leqslant \frac{1}{\lambda_{0}(p+1)} \int_{0}^{b} r(x)\left|u^{\prime}(x)\right|^{p+1} d x,
\end{aligned}
$$

valid for any $u$ such that $u(x)=\int_{0}^{x} u^{\prime} d t$ for $x \in[0, b]$, where $\lambda_{0}$ is the smallest eigenvalue of the boundary value problem (2.5.1). Equality is attained in (2.5.2) by the eigenfunctions corresponding to $\lambda_{0}$. (See also the comments in Zbl. Math. 173 (1969), p. 57, by P. R. Beesack, related to the paper [11].) We also note that there is only one eigenvalue $\lambda$ of (2.5.1) as pointed out later by one of the authors. This means that there is no loss of generality in setting $\lambda=1$ in (2.5.1), and $\lambda_{0}=1$ in (2.5.2).

In the present chapter we have generalized the above result in two aspects:

(1) We considered two parameters $p, q$ rather than one parameter $p$ only. (Compare (2.5.1), (2.5.2) with (2.2.1) and (2.2.4).) (2) We consider the best possible constants when the solution of (2.2.1) is not admissible. The second point is more important and was dealt with in $\S 2.3$ by unifying Beesack's method with Benson's approach. The result in this section is not covered in either [11] or [12], because neither in [11] nor in [12], is it possible to obtain the best 
possible constant when the solution of a certain boundary value problem is not admissible. (This shows the fruitfulness of unifying the above-mentioned two methods.)

In [9], some inequalities of the form dealt with in $\$ 2.2$ were obtained by using Holder's inequality, and even with a broader range of values of the parameters $p, q$. That is, inequalities of the type (2.2.4) were obtained in [9] for all $p, q$ such that $p q>0$ and either $p+q \geqslant 1$ or $p+q<0$, instead of either $p \geqslant 0, q \geqslant 1$ or $p<0, q<0$ as in Theorem 2.1. Moreover, in [9], a reversed inequality of the type (2.2.4) was also obtained in case $p<0$, $q \geqslant 1, p+q>1$, or $p>0, p+q<0$. (We also note that the reversed inequality of a certain type was dealt with, for the first time, in [5], where the author considered the inequalities of the Hardy type, systematically and uniformly.) However, in [9] the inequalities were obtained without reference to any differential boundary value problem, and most of the inequalities obtained there were not sharp.

It may be of interest to note that the sharp case of [9, Theorem 1] is included in our Theorem 2.1 in case $p \geqslant 0, q \geqslant 1$, or $p<0, q<0$.

It may also be of interest that the results in Chapter 2 are comparable with those in [12] in some aspects. In order to state the various inclusion relations we first note that in [12] the following theorem was proved from a functional analysis point of view.

THEOREM (D. W. BOYD). Suppose that $r_{1}, s_{1} \in C^{1}(a, b)$, that $s_{1}(x)>0$ a.e. and $r_{1}(x)>0$ for $a<x<b$, that $k, p, q$ are real numbers satisfying $p>0, k>1,0 \leqslant q<k$, and that the operator $T_{1}$ defined by

$$
T_{1} f(x)=\left[s_{1}(x)\right]^{1 / p}\left[r_{1}(x)\right]^{-1 / p} \int_{a}^{x} f(t) d t
$$

is compact from $L_{r_{1}}^{k} \rightarrow L_{r_{1}}^{t}$ with $t=p k /(k-q)$. (The symbol $L_{r_{1}}^{k}$ denotes the set of functions with $\|f\|_{k}=\left\{\int_{a}^{b} r_{1}|f|^{k} d x\right\}^{1 / k}<\infty$; since $k>1, L_{r_{1}}^{k}$ is a Banach space.) Then the following eigenvalue problem $(\mathrm{P})$ has solutions $(y, \lambda)$ with $y \in C^{2}(a, b)$ and $y(x)>0, y^{\prime}(x)>0$ in $(a, b)$.

(1) $\frac{d}{d x}\left[k \lambda\left(y^{\prime}\right)^{k-1} r_{1}-q y^{p}\left(y^{\prime}\right)^{q-1} s_{1}\right]+p y^{p-1}\left(y^{\prime}\right)^{q} s_{1}=0$,

(P) (2) $\lim _{x \rightarrow a+} y(x)=0$ and $\lim _{x \rightarrow b-}\left[k \lambda\left(y^{\prime}\right)^{k-1} r_{1}-q y^{p}\left(y^{\prime}\right)^{q-1} s_{1}\right]=0$,

(3) $\left\|y^{\prime}\right\|_{k}=1$.

There is a largest value $\lambda$ such that $(\mathrm{P})$ has a solution and if $\lambda^{*}$ denotes this value, then for any $f \in L_{r_{1}}^{k}$, 


$$
\int_{a}^{b}\left|\int_{a}^{x} f\right|^{p}|f|^{q} s_{1}(x) d x \leqslant \frac{k \lambda^{*}}{p+q}\left(\int_{a}^{b}|f|^{k} r_{1}(x) d x\right)^{(p+q) / k}
$$

Equality holds in (2.5.4) if and only if $f=c y^{\prime}$ a.e. where $y$ is a solution of (P) corresponding to $\lambda=\lambda^{*}$, and $c$ is any constant.

(Perhaps this an appropriate place to emphasize that in the papers [2]-[5], Beesack, for the first time, made use of the Euler-Lagrange differential equations associated with some extremal problems to investigate integral inequalities by integral identities; he found that when the solution of a differential equation is an admissible function for a certain type of inequality, then equality is actually attained by this very solution. Moreover, if this solution is not admissible, he still made use of the differential equation to discuss the sharpness, that is, the best possible constants of the inequalities. In this chapter we have used his method for the first time to duscuss the sharpness of Opial type inequalities.)

Now, from (2.5.4) we see that the case $k=p+q$, of Boyd's theorem, is the only one which is comparable with the sharp case of Theorem 2.1 (with $p>0, q \geqslant 1$ ). Actually, the latter is included in the former, and Theorem 2.1 includes those cases of Boyd's theorem for which $r_{1}, s_{1}$ are continuous and strictly positive, on a compact interval $[a, b]$.

Finally, we note that Boyd's theorem does not include the case $p<0$, $q<0$ of Theorem 2.1, that our methods are quite elementary whereas Boyd's are not, and that the $\$ 2.3$ includes sharp inequalities when the solution $y$ of the boundary value problem is not admissible (because $\int_{a}^{b} r\left(y^{\prime}\right)^{(p+q) / q} d x=\infty$ ) whereas (as pointed out in [12, p. 383]) Boyd's method cannot handle such inequalities. On the other hand, in Boyd's theorem, the existence of a solution of the boundary value problem is a conclusion of the theorem, rather than a hypothesis. In the last chapter, §4.5, Boyd's theorem will be considered again, when it is compared with the results of Chapters 3 and 4.

\section{Chapter 3. On a Class of New Inequalities}

3.1. Introduction. In this chapter we are concerned with a systematic and uniform treatment of some analogues and extensions of Hardy's inequality for integrals and of Opial's inequality.

In 1968, Beesack and Das [9] considered integral inequalities of the form

$$
\int_{a}^{b} s|u|^{p}\left|u^{\prime}\right|^{q} d x \leqslant k(p, q) \int_{a}^{b} r\left|u^{\prime}\right|^{p+q} d x
$$

(or with $\leqslant$ replaced by $\geqslant$ ), where $r, s$ are nonnegative measurable functions on $I=(a, b)$, and $u$ is absolutely continuous on $I$ with either $u(a)=0$, or $u(b)=0$, or both. 
The main extensions dealt with here are all integral inequalities of the form:

$$
\begin{gathered}
\left(\int_{a}^{b} W|u|^{p}\left|u^{\prime}\right|^{q} d x\right)^{k /(p+q)}+\left(\frac{q}{p+q}\right)^{k /(p+q)} C^{-1} \int_{a}^{b} S|u|^{k} d x \\
\leqslant\left(\frac{q}{p+q}\right)^{k /(p+q)} C^{-1} \int_{a}^{b} R\left|u^{\prime}\right|^{k} d x
\end{gathered}
$$

where either $u(a)=0$, or $u(b)=0$.

One of the most interesting things about the above inequality is that when the first term on the left side is replaced by zero, it actually includes Theorem 3.1.1 in [5] (see also (1.1) and (1.2)). Since the terms and factors on the left side of (3.1.2) are all nonnegative, (3.1.2) is an improvement of the results both in [5] and [9].

We also note that in a recent paper [12], Boyd obtained best constants in a class of integral inequalities similar to (3.1.2), but with the second term on the left side of (3.1.2) replaced by zero. (A comparison of our results with those of Boyd will also be given in §4.5.)

3.2. The main results. We consider the following nonlinear differential boundary value problem.

(a) $\left[r\left(y^{\prime}\right)^{(k-q) / q}\right]^{\prime}+s y^{(k-q) / \dot{q}}=0$,

(b) $\lim _{x \rightarrow a+} r\left(y^{\prime} / y\right)^{(k-q) / q} y^{k / q}=0$,

(c) $\left\{\begin{array}{l}\lim _{x \rightarrow b_{-}} r\left(y^{\prime} / y\right)^{(k-q) / q}=C(0<C<\infty), \\ \lim _{x \rightarrow b_{-}}\left[r\left(y^{\prime} / y\right)^{(k-q) / q}-C\right] y^{k / q}=0,\end{array}\right.$

(d) $r\left(y^{\prime} / y\right)^{(k-q) / q}\left(\int_{a}^{x} r^{-q /(k-q)} d t\right)^{(k-q) / q}=O(1)$ as $x \rightarrow a+$,

(e) $\int_{a}^{b} r\left(y^{\prime}\right)^{k / a} d x<\infty$.

Here, $r$ is positive in $C(a, b)$ ( $a$ or $b$ or both may be infinite), $s$ is nonnegative in $C(a, b)$. We shall assume that (3.2.1) has a solution $y$ such that $y=$ $\int_{a}^{x} y^{\prime} d t$ with $y^{\prime}(x)>0$ on $(a, b)$, and $y(b)<\infty$ in case $k / q<0$.

By setting $h=\left(y^{\prime} / y\right)^{(k-q) / q}$, from (3.2.1a) we see that $h$ satisfies the Riccati-like equation

$$
\frac{k-q}{q} r h^{k /(k-q)}+(r h)^{\prime}=-s(x), \quad x \in(a, b) .
$$


We note that, in case $(k-q) / q>0$, then by (3.2.2) $r h$ is a decreasing function on $(a, b)$, so that $C$ in (3.2.1c) is finite automatically in this case.

THEOREM 3.1. Suppose that the boundary value problem (3.2.1) has a solution $y(x)$ such that $y(x)=\int_{a}^{x} y^{\prime} d t$ with $y^{\prime}(x)>0$ on $(a, b)$ and $y(b)<$ $\infty$ in case $k / q<0$. Let $w$ be any positive measurable function on $(a, b)$ such that $\int_{a}^{x} w^{-1} d t<\infty$, and define $W, R$ and $S$ on $(a, b)$ by

$$
\begin{aligned}
& W(x)=[w(x)]^{q-1}\left(\int_{a}^{x} w^{-1} d t\right)^{p(1-q) / q}, \\
& R(x)=r(x)[w(x)]^{k(q-1) / q}, \\
& S(x)=s(x)\left(\int_{a}^{x} w^{-1} d t\right)^{k(1-q) / q} .
\end{aligned}
$$

Finally, let $u$ be any locally absolutely continuous function on $(a, b)$ with $u(x)=\int_{a}^{x} u^{\prime} d t$ such that $\int_{a}^{b} R\left|u^{\prime}\right|^{k} d x<\infty$. Then

$$
\begin{gathered}
\left(\int_{a}^{b} W|u|^{p}\left|u^{\prime}\right|^{q} d x\right)^{k /(p+q)}+\left(\frac{q}{p+q}\right)^{k /(p+q)} C^{-1} \int_{a}^{b} S|u|^{k} d x \\
\leqslant\left(\frac{q}{p+q}\right)^{k /(p+q)} C^{-1} \int_{a}^{b} R\left|u^{\prime}\right|^{k} d x
\end{gathered}
$$

valid for $p>0, k>q \geqslant 1$, where $C$ is defined in (3.2.1c). If, in addition, $u^{\prime}$ does not change sign on $(a, b)$, then (3.2.4) is still valid for all $p, q, k$ such that either $p<0, k<q<0$, or $p>0, k<0<q \leqslant 1$, if $\int_{a}^{b} w^{-1} d t<\infty$ and $|u(b)|<\infty$ in the latter case.

Equality holds in (3.2.4) if and only if

$$
\begin{aligned}
& u=k_{1} y \quad\left(k_{1} \text { a constant }\right), \\
& w=k_{2}\left(y^{\prime}\right)^{-1} \quad\left(k_{2}>0, a \text { constant }\right) \quad(q \neq 1), \\
& \text { or } u=k_{1} y, \text { if } q=1 \text {. }
\end{aligned}
$$

In order to prove this theorem, we require the following lemma.

LEMMA 3.1. Let $k / q>1$ or $k<0<q \leqslant 1$ and suppose that $\int_{a}^{b} R\left|u^{\prime}\right|^{k} d x<\infty$ and $\left(\int_{a}^{x} r^{-q /(k-q)} d t\right)^{(k-q) / q} r(x) h(x)=O(1)$ as $x \rightarrow a+$. Then, $\int_{a}^{x} w^{q-1}\left|u^{\prime}\right|^{q} d t<\infty$ for $a \leqslant x<b$, and

$$
\lim _{x \rightarrow a+}\left(\int_{a}^{x} w^{q-1}\left|u^{\prime}\right|^{q} d t\right)^{k / q} r(x) h(x)=0 .
$$

In the case $k<0<q \leqslant 1$, we also have $\int_{a}^{b} w^{q-1}\left|u^{\prime}\right|^{q} d x<\infty$, if $\int_{a}^{b} w^{-1} d x<$ $\infty$ and $|u(b)|<\infty$. 
Proof. We note that all hypotheses in the lemma are stated in Theorem 3.1; the proof of this lemma may be completed by using Hölder's inequality.

Proof OF THEOREM 3.1. First we note that from (1.6), with $p$ replaced by $k / q$, we have for $k / q>1$ (or $k / q<0$ ), and $a<\alpha<\beta<b$,

$$
\begin{aligned}
\int_{\alpha}^{\beta}\left[Q\left(v^{\prime}\right)^{k / q}+\right. & \left.((k-q) / q)\left(G_{v}\right)^{k /(k-q)} Q^{q /(q-k)}+(k / q) G_{x}\right] d x \\
& \geqslant\left.(k / q) G(v, x)\right|_{\alpha} ^{\beta} .
\end{aligned}
$$

By (1.8) equality holds in (3.2.7) if and only if the differential equation

$$
v^{\prime}=\left(G_{v}\right)^{q /(k-q)} Q^{q /(q-k)}
$$

is satisfied almost everywhere.

Now set

$$
\begin{aligned}
& z=\int_{a}^{x} w^{q-1}\left|u^{\prime}\right|^{q} d t \quad \text { (which exists by Lemma 3.1), } \\
& v=\frac{q}{k}\left(\frac{p+q}{q}\right)^{k /(p+q)}\left(\int_{a}^{x} w^{q-1}\left|u^{\prime}\right|^{q} z^{p / q} d t\right)^{k /(p+q)}=\frac{q}{k} z^{k / q} .
\end{aligned}
$$

(Note $(p+q) / q>0$.) Also, setting $Q=r z^{k(q-k) / q^{2}}, G=v(r h)$, we obtain from (3.2.7), for $a<\alpha<\beta<b$,

$$
\begin{aligned}
& \int_{\alpha}^{\beta}\left(R\left|u^{\prime}\right|^{k}+\left[\frac{k-q}{q} r h^{k /(k-q)}+(r h)^{\prime}\right] z^{k / q}\right) d x \\
& \geqslant\left.\left(\frac{p+q}{q}\right)^{k /(p+q)} r(x) h(x)\left(\int_{a}^{x} w^{q-1}\left|u^{\prime}\right|^{q} z^{p / q} d t\right)^{k /(p+q)}\right|_{\alpha} ^{\beta} .
\end{aligned}
$$

By using (3.2.2) we have, from (3.2.9),

$$
\begin{aligned}
\int_{\alpha}^{\beta} R\left|u^{\prime}\right|^{k} d x \geqslant 2 \int_{\alpha}^{\beta} s z^{k / q} d x & +\left(\frac{p+q}{q}\right)^{k /(p+q)} r(x) h(x) \\
& \times\left.\left(\int_{a}^{x} w^{q-1}\left|u^{\prime}\right|^{q} z^{p / q} d t\right)^{k /(p+q)}\right|_{\alpha} ^{\beta} \\
= & \int_{\alpha}^{\beta} s z^{k / q} d x+\left(\frac{p+q}{q}\right)^{k /(p+q)} r(\beta) h(\beta) \\
& \times\left(\int_{a}^{\beta} w^{q-1}\left|u^{\prime}\right|^{q} z^{p / q} d t\right)^{k /(p+q)} \\
& -\left(\frac{p+q}{q}\right)^{k /(p+q)} r(\alpha) h(\alpha)\left(\int_{a}^{\alpha} w^{q-1}\left|u^{\prime}\right|^{q} d t\right)^{k / q}
\end{aligned}
$$


On noting that $s(x) \geqslant 0$ so that the integral $\int_{a}^{\beta} s z^{k / q} d x$ exists, we obtain from (3.2.10) by letting $\alpha \rightarrow a+$, on using (3.2.6),

$$
\begin{gathered}
\left(\frac{p+q}{q}\right)^{k /(p+q)} r(\beta) h(\beta)\left(\int_{a}^{\beta} w^{q-1}\left|u^{\prime}\right|^{q} z^{p / q} d t\right)^{k /(p+q)} \\
+\int_{a}^{\beta} s z^{k / q} d t \leqslant \int_{a}^{f} R\left|u^{\prime}\right|^{k} d x \geqslant
\end{gathered}
$$

Now by letting $\beta \rightarrow b-$ we finally get, on noting the first part of (3.2.1c),

$$
\begin{gathered}
\left(\int_{a}^{b} w^{q-1}\left|u^{\prime}\right|^{q} z^{p / q} d x\right)^{k /(p+q)}+\left(\frac{q}{p+q}\right)^{k /(p+q)} C^{-1} \int_{a}^{b} s z^{k / q} d x \\
\leqslant\left(\frac{q}{p+q}\right)^{k /(p+q)} C^{-1} \int_{a} R\left|u^{\prime}\right|^{k} d x
\end{gathered}
$$

We note that when $p>0, k<0<q \leqslant 1$, we have $\int_{a}^{b} w^{-1} d x<\infty$ and $|u(b)|<\infty$. Hence, by Lemma 3.1 we have $\int_{a}^{b} w^{q-1}\left|u^{\prime}\right|^{q} d x<\infty$, which assures us that the integral $\int_{a}^{b} w^{q-1}\left|u^{\prime}\right|^{q} z^{p / q} d x$ in (3.2.12) exists (finite).

Now for $p>0$, by using Holder's inequality, with indices $q$ and $q /(q-1)$, we have, for $q>1$,

$$
\left(\int_{a}^{x} w^{-1} d t\right)^{p(1-q) / q}|u|^{p} \leqslant z^{p / q} \quad \text { (see (2.2.13)). }
$$

Hence,

$$
\int_{a}^{b} W|u|^{p}\left|u^{\prime}\right|^{q} d x \leqslant \int_{a}^{b} w^{q-1}\left|u^{\prime}\right|^{q} z^{p / q} d x
$$

We note that if $q=1$, then (3.2.13) and (3.2.14) are still valid, and that if $u^{\prime}$ does not change sign on $(a, b)$, then (3.2.13) is also still valid for $p<0, q<0$. Thus, for $p<0, k<q<0,(3.2 .14)$ is still valid, if $u^{\prime}$ does not change sign on $(a, b)$. We also note that if $u^{\prime}$ does not change sign on $(a, b)$ and $p>0,0<q \leqslant 1$, then we have

$$
\begin{gathered}
\left(\int_{a}^{x} w^{-1} d t\right)^{p(1-q) / q}|u|^{p} \geqslant z^{p / q} \\
\int_{a}^{b} W|u|^{p}\left|u^{\prime}\right|^{q} d x \geqslant \int_{a}^{b} w^{q-1}\left|u^{\prime}\right|^{q} z^{p / q} d x .
\end{gathered}
$$

Finally we note that the integral $\int_{a}^{b} W|u|^{p}\left|u^{\prime}\right|^{q} d x$ in (3.2.14a) exists (finite), since in this case $(p>0, k<0<q \leqslant 1)$ all three conditions: $u^{\prime}$ is of one sign; $|u(b)|<\infty$; and $\int_{a}^{b} w^{-1} d x<\infty$; are assumed; and one obtains $\int_{a}^{b} W|u|^{p}\left|u^{\prime}\right|^{q} d x \leqslant$ $K \int_{a}^{b} w^{q-1}\left|u^{\prime}\right|^{q} d x<\infty$ by Lemma 3.1. 
Now, from (3.2.13) we have for $k / p>0$,

$$
\left(\int_{a}^{x} w^{-1} d t\right)^{k+(1-q) / q}|u|^{k} \leqslant z^{k / q} .
$$

Hence,

$$
\int_{a}^{b} S|u|^{k} d x \leqslant \int_{a}^{b} s z^{k / a} d x
$$

In case $p>0, k<0<q \leqslant 1,(3.2 .15)$ and hence (3.2.16) are still valid. This follows from (3.2.13a) and the fact that $k / p<0$ in this case.

Combining (3.2.12), (3.2.14) and (3.2.16), we have thus proved (3.2.4) for $p>0, k>q \geqslant 1$ or $p<0, k<q<0$ on noting that $k /(p+q)>0$ in these cases. Similarly, combining (3.2.12), (3.2.14a) and (3.2.16), we obtain (3.2.4) for $p>0, k<0<q \leqslant 1$, on noting that $k /(p+q)<0$ in the present case.

Although the details of proving the equality conditions are quite long, the key for proving these conditions is similar to that given at the end of the proof of Theorem 2.1.

Since we shall not establish another theorem with $u(b)=0$ (see also Remark 2.2), we state the following corollary without proof, which is based on both $u(a)=0$ (Theorem 3.1) and the corresponding case with $u(b)=0$.

COROLLARY 3.1. Let $u$ be locally absolutely continuous on $(a, X]$ and $[X, b)$ with $u(x)=\int_{a}^{x} u^{\prime} d t, a \leqslant x \leqslant X, u(x)=-\int_{x}^{b} u^{\prime} d t, X \leqslant x \leqslant b$, where $X$ is the unique solution of

$$
\int_{a}^{X} r^{-q / p} d t=\int_{X}^{b} r^{-q / p} d t\left(\int_{a}^{b} r^{-q / p} d t<\infty\right) .
$$

Here, $r(x)$ is positive and measurable on $(a, X),(X, b)$ respectively. Let $w$ be any positive and measurable function on $(a, b)$ such that $\int_{a}^{x} w^{-1} d t<\infty$ for $x \in(a, X), \int_{x}^{b} w^{-1} d t<\infty$ for $x \in(X, b)$, and define $W, R$ on $(a, b)$ by

$$
\begin{aligned}
& W(x)= \begin{cases}{[w(x)]^{q-1}\left(\int_{a}^{x} w^{-1} d t\right)^{p(1-q) / q},} & x \in(a, X), \\
{[w(x)]^{q-1}\left(\int_{x}^{b} w^{-1} d t\right)^{p(1-q) / q},} & x \in(X, b),\end{cases} \\
& R(x)=r(x)[w(x)]^{(p+q)(q-1) / q}, x \in(a, b) .
\end{aligned}
$$

Then, if $\int_{a}^{b} R\left|u^{\prime}\right|^{p+q} d x<\infty$,

$$
\int_{a}^{b} W|u|^{p}\left|u^{\prime}\right|^{q} d x \leqslant \frac{q}{p+q}\left(\int_{a}^{x} r^{-q / p} d t\right)^{p / q} \int_{a}^{b} R\left|u^{\prime}\right|^{p+q} d x
$$


Here, either $p>0, q \geqslant 1$, or $p<0, q<0$, if $u^{\prime}$ is of one sign on $(a, X)$ and $(X, b)$ respectively in the latter case.

Equality holds in (3.2.19) if and only if

$$
\begin{aligned}
& u(x)= \begin{cases}A \int_{a}^{x} r^{-q / p} d t, & x \in(a, X), \\
B \int_{x}^{b} r^{-q / p} d t, & x \in(X, b),\end{cases} \\
& w(x)=C^{*}[r(x)]^{q / p}, \quad x \in(a, b)(q \neq 1) .
\end{aligned}
$$

Here, $A, B, C^{*}$ are constants. If $q=1, w(x)$ in (3.2.20) is omitted.

REMARK 3.1. In (3.2.19) setting $a=0<b<\infty, W=R \equiv 1$, and $p=$ $q=1$, we obtain Opial's original inequality.

3.3. The sharpness of the inequalities when $y$ is not admissible. We are going to deal with those cases where the inequality (3.2.4) is best possible, but equality is only attained in the trivial case $u \equiv 0$ (or never in case $p<0$, $k<q<0$ or $p>0, k<0,0<q \leqslant 1)$. We first note that when $w=\left(y^{\prime}\right)^{-1}$, (3.2.3) and (3.2.4) reduce to

$$
W=y^{p(1-q) / q}\left(y^{\prime}\right)^{1-q},
$$

$$
\begin{aligned}
& R=r\left(y^{\prime}\right)^{k(1-q) / q} \\
& S=s y^{k(1-q) / q} \\
&\left(\int_{a}^{b} y^{p(1-q) / q}\left(y^{\prime}\right)^{1-q}|u|^{p}\left|u^{\prime}\right|^{q} d x\right)^{k /(p+q)} \\
&+(q /(p+q))^{k /(p+q)} C^{-1} \int_{a}^{b} s y^{k(1-q) / q}|u|^{k} d x \\
& \leqslant(q /(p+q))^{k /(p+q)} C^{-1} \int_{a}^{b} r\left(y^{\prime}\right)^{k(1-q) / q}\left|u^{\prime}\right|^{k} d x
\end{aligned}
$$

We want to show that the constant $(q /(p+q))^{k /(p+q)} C^{-1}$ is the best possible when the left side of (3.3.2) is unchanged. There are three cases $p>0, k>q \geqslant 1$, and $p<0, k<q<0$ and $p>0, k<0<q \leqslant 1$. We first consider $p>0, k>q \geqslant 1$. The hypotheses as stated in Theorem 3.1 are mainly unchanged except that instead of (3.2.1) we now assume the following nonlinear differential boundary value problem 
(a) $\left(r\left(y^{\prime}\right)^{(k-q) / q}\right)^{\prime}+s y^{(k-q) / q}=0$,

(b) $\varliminf_{x \rightarrow a^{+}} r\left(y^{\prime} / y\right)^{(k-q) / q} y^{k / q}<\infty$,

(c) $\left\{\begin{array}{l}\lim _{x \rightarrow b_{-}^{-}} r\left(y^{\prime} / y\right)^{(k-q) / a}=C \quad(0<C<\infty), \\ \lim _{x \rightarrow b_{-}} y(x)=D<\infty\end{array}\right.$

(d) $\left(\int_{a}^{x} r^{-q /(k-q)} d t\right)^{(k-q) / q} r\left(y^{\prime} / y\right)^{(k-q) / q}=O(1) \quad$ as $x \rightarrow a+$,

(e) $\int_{a}^{b} r\left(y^{\prime}\right)^{k / a} d x=\infty$,

has a solution $y(x)=\int_{a}^{x} y^{\prime} d t$ such that $y^{\prime}(x)>0$ on $(a, b)$. (We note that $y(x)=\int_{a}^{x} y^{\prime} d t$ assures us that we can get (3.3.1) from (3.2.3), and that (3.3.3b) is weaker than (3.2.1b), whereas (3.3.3c) is stronger than (3.2.1c). Only (3.3.3a), the first of (3.3.3c), and (3.3.3d) were used to establish the validity of (3.3.2) for all admissible functions $u$.) Under these assumptions (3.3.2) is still sharp, that is, the constant $(q /(p+q))^{k /(p+q)} C^{-1}$ is best possible when the left side of (3.3.2) is unchanged, although equality holds in (3.3.2) if and only if $u \equiv 0$.

Now to prove this, we take

$$
\begin{aligned}
u^{\prime} & =0, & a \leqslant x \leqslant a^{\prime}, \\
& =y^{\prime}, \quad & a^{\prime}<x<b^{\prime}, \\
& =0, & b^{\prime} \leqslant x \leqslant b,
\end{aligned}
$$

where $a^{\prime}, b^{\prime}$ will be determined later. Then $u(x)=y(x)-y\left(a^{\prime}\right)$ for $a^{\prime}<$ $x<b^{\prime}$ and, for $k>1$,

$$
\begin{aligned}
(u(x))^{k} & =(y(x))^{k}\left(1-\frac{y\left(a^{\prime}\right)}{y(x)}\right)^{k} \\
& >(y(x))^{k}\left(1-k \frac{y\left(a^{\prime}\right)}{y(x)}\right), \quad a^{\prime}<x<b^{\prime} .
\end{aligned}
$$

This inequality is a special case of (1.4) with $p$ replaced by $k$, obtained by setting $s=1-\left(y\left(a^{\prime}\right) / y(x)\right), t=1$.

Now by using (3.3.1), (3.3.4) and (3.3.5), we have 


$$
\begin{aligned}
& \left(\int_{a^{\prime}}^{b^{\prime}} W|u|^{p}\left|u^{\prime}\right|^{q} d x\right)^{k /(p+q)}+(q /(p+q))^{k /(p+q)} C^{-1} \int_{a^{\prime}}^{b^{\prime}} S|u|^{k} d x \\
& >(q /(p+q))^{k /(p+q)} C^{-1} \int_{a^{\prime}}^{b^{\prime}} S|u(x)|^{k} d x \\
& =(q /(p+q))^{k /(p+q)} C^{-1} \int_{a^{\prime}}^{b^{\prime}} s y^{k(1-q) / q} u^{k} d x \\
& >(q /(p+q))^{k /(p+q)} C^{-1}\left(\int_{a^{\prime}}^{b^{\prime}} s y^{k / q} d x-k y\left(a^{\prime}\right) \int_{a^{\prime}}^{b^{\prime}} s y^{(k-q) / q} d x\right)
\end{aligned}
$$

Now by (3.3.3a) we have

and

$$
\begin{aligned}
\int_{a^{\prime}}^{b^{\prime}} s y^{k / q} d x & =-\int_{a^{\prime}}^{b^{\prime}}\left(r\left(y^{\prime}\right)^{(k-q) / q}\right)^{\prime} y d x \\
& =-\left.r y\left(y^{\prime}\right)^{(k-q) / q}\right|_{a^{\prime}} ^{b^{\prime}}+\int_{a^{\prime}}^{b^{\prime}} r\left(y^{\prime}\right)^{k / q} d x,
\end{aligned}
$$

$$
\begin{aligned}
\int_{a^{\prime}}^{b^{\prime}} s y^{(k-q) / q} d x & =-\int_{a^{\prime}}^{b^{\prime}}\left(r\left(y^{\prime}\right)^{(k-q) / q}\right)^{\prime} d x \\
& =-\left.r\left(y^{\prime}\right)^{(k-q) / q}\right|_{a^{\prime}} ^{b^{\prime}} \\
& =-\left.r h y^{(k-q) / q}\right|_{a^{\prime}} ^{b^{\prime}}
\end{aligned}
$$

On noting (3.3.7), (3.3.8), and the definition of $u^{\prime}$ in (3.3.4), from (3.3.6) it follows that

$$
\left(\int_{a}^{b} W|u|^{p}\left|u^{\prime}\right|^{q} d x\right)^{k /(p+q)}+(q /(p+q))^{k /(p+q)} C^{-1} \int_{a}^{b} S|u|^{k} d x
$$

$$
\begin{aligned}
(3.3 .9)> & -(q /(p+q))^{k /(p+q)} C^{-1}\left(\left.r y\left(y^{\prime}\right)^{(k-q) / q}\right|_{a^{\prime}} ^{b^{\prime}}-\int_{a}^{b} r\left(y^{\prime}\right)^{k(1-q) / q}\left|u^{\prime}\right|^{k} d x\right) \\
& +\left.(q /(p+q))^{k /(p+q)} k C^{-1} y\left(a^{\prime}\right) r\left(y^{\prime}\right)^{(k-q) / q}\right|_{a^{\prime}} ^{b^{\prime}}
\end{aligned}
$$

Hence, for any $\delta>0$ we have

$$
\left(\int_{a}^{b} W|u|^{p}\left|u^{\prime}\right|^{q} d x\right)^{k /(p+q)}+(q /(p+q))^{k /(p+q)} C^{-1} \int_{a}^{b} S|u|^{k} d x
$$

$$
\begin{aligned}
& >(1-\delta)(q /(p+q))^{k /(p+q)} C^{-1} \int_{a}^{b} R\left|u^{\prime}\right|^{k} d x \\
& =(1-\delta)(q /(p+q))^{k /(p+q)} C^{-1} \int_{a}^{b} r\left(y^{\prime}\right)^{k(1-q) / q}\left|u^{\prime}\right|^{k} d x,
\end{aligned}
$$

provided 


$$
\begin{aligned}
& \delta(q /(p+q))^{k /(p+q)} C^{-1} \int_{a^{\prime}}^{b^{\prime}} r\left(y^{\prime}\right)^{k / q} d x \\
& \quad>\left.(q /(p+q))^{k /(p+q)} C^{-1} r(x) h(x)(y(x))^{k / q}\left(1-. k y\left(a^{\prime}\right)(y(x))^{-1}\right)\right|_{a^{\prime}} ^{b^{\prime}}
\end{aligned}
$$

Now, the terms on the right side of (3.3.11) are bounded as $a^{\prime} \rightarrow a+, b^{\prime} \rightarrow$ $b-$, respecitively, by using (3.3.3b) and (3.3.3c) together with the fact that $y(x)$ is increasing on $(a, b)$. Hence, by taking $b^{\prime}$ sufficiently close to $b$, and $a^{\prime}$ appropriately close to $a$, the inequality (3.3.11) follows from (3.3.3e), completing the proof of sharpness in this case.

REMARK 3.2. In order to get the first inequality in (3.3.6) the Opial type term was dropped.

We now consider the case $k<0$ (that is, the remaining two cases $p>0$, $k<0<q \leqslant 1$ and $p<0, k<q<0$ ). These cases are characterized by $k<0$, and the following proof is suitable for both cases. When $k<0$, equality in (3.2.4) never occurs in view of our requirement $u^{\prime}>0$, and we must modify the procedure used before. The hypotheses are precisely the same as those in (3.3.3) except that the condition (3.3.3b) is now replaced by

$$
\lim _{x \rightarrow a+} r(x)\left(y^{\prime}(x) / y(x)\right)^{(k-q) / q}(y(x))^{(k-q) / q}<\infty .
$$

(We note that (3.3.12) is a stronger requirement than either (3.2.1b), or (3.3.3b). Also, by (3.3.8) we see that $r h y^{(k-q) / q}$ is monotone decreasing on $(a, b)$, and hence the limit in question exists, finite or infinite.)

Now, we set

$$
\begin{aligned}
u^{\prime}(x) & =g(x), & & a \leqslant x \leqslant a^{\prime}, \\
& =y^{\prime}(x), & & a^{\prime}<x<b^{\prime}, \\
& =g(x), & & b^{\prime} \leqslant x \leqslant b .
\end{aligned}
$$

Here $a^{\prime}, b^{\prime}$ are to be determined, and $g(x)>0$ is the derivative of any (fixed) admissible function. The remaining part of the proof is more or less similar to that in the case $k>1$. (See also the last part of $\S 2.3$.)

REMARK 3.3. We emphasize that in this section we need the condition that the function $s$ is not identically zero on $(a, b)$; otherwise, all proofs in this section will fail to be true, leaving the question whether the constant is still best possible undecided.

3.4. Some examples. Let $\alpha<1,0 \leqslant \beta<1$ with $1-\beta \neq k / q$, and $-\infty<$ $a<b<\infty$. Suppose that $p>0, k>q \geqslant 1$, or $p<0, k<q<0$, or $p>0$, $k<0<q \leqslant 1$. Setting 


$$
\begin{aligned}
& r(x)=(x-a)^{\alpha((k-q) / q+\beta)-\beta}, \\
& s(x)=(1-\alpha)^{k / q} \beta(x-a)^{(\alpha-1)(k / q+\beta)-\alpha, \text { and }} \\
& y(x)=(1-\alpha)^{-1}(x-a)^{1-\alpha,}
\end{aligned}
$$

we see that $y$ satisfies $(3.2 .1)$ on $(a, b)$ with

$$
C=(1-\alpha)^{(k-q) / q}(b-a)^{(\alpha-1)((k-q) / q+\beta)}
$$

in (3.2.1c). Now we set $w=(x-a)^{\alpha}$, so that $w=\left(y^{\prime}\right)^{-1}$. All hypotheses of Theorem 3.1 are satisfied. From (3.2.4) we have, after simplification,

$$
\begin{aligned}
& (1-\alpha)^{k(p+1) /(p+q)-1}\left(\int_{a}^{b}(x-a)^{(q-1)(\alpha(p+q)-p) / q}|u|^{p}\left|u^{\prime}\right|^{q} d x\right)^{k /(p+q)} \\
& +\left(\frac{q}{p+q}\right)^{k /(p+q)}(1-\alpha)^{k} \beta(b-a)^{(1-\alpha)((k-q) / q+\beta)} \int_{a}^{b}(x-a)^{(\alpha-1)(k+\beta)-\alpha}|u|^{k} d x \\
& \leqslant\left(\frac{q}{p+q}\right)^{k /(p+q)}(b-a)^{(1-\alpha)((k-q) / q+\beta)} \int_{a}^{b}(x-a)^{\alpha(k-1)+(\alpha-1) \beta}\left|u^{\prime}\right|^{k} d x,
\end{aligned}
$$

valid for any $u$ such that $u=\int_{a}^{x} u^{\prime} d t$ for every $x \in(a, b)$, and $\int_{a}^{b}(x-a)^{\alpha(k-1)+(\alpha-1) \beta}\left|u^{\prime}\right|^{k} d x<\infty$ and for any $p, q, k$ such that $p>0$, $k>q \geqslant 1$. If, in addition, $u^{\prime}$ does not change sign on $(a, b)$, then (3.4.1) is still valid for any $p, q, k$ such that $p<0, k<q<0$. Finally, if the admissible function $u$ is further restricted to satisfy the condition $|u(b)|<\infty$, then (3.4.1) is also valid for any $p, q, k$ such that $p>0, k<0<q \leqslant 1$. Equality holds in (3.4.1) if and only if $u=k_{1}(x-a)^{1-\alpha}$.

EXAMPLE 3.1. Taking $\alpha=0$ in (3.4.1) we have

$$
\begin{aligned}
& \left(\int_{a}^{b}(x-a)^{p(1-q) / q}|u|^{p}\left|u^{\prime}\right|^{q} d x\right)^{k /(p+q)} \\
& \quad+(q /(p+q))^{k /(p+q)} \beta(b-a)^{((k-q) / q)+\beta} \int_{a}^{b}(x-a)^{-(k+\beta)}|u|^{k} d x \\
& \quad \leqslant(q /(p+q))^{k /(p+q)}(b-a)^{((k-q) / q)+\beta} \int_{a}^{b}(x-a)^{-\beta}\left|u^{\prime}\right|^{k} d x,
\end{aligned}
$$

valid for any $u$ stated below (3.4.1) with $\alpha=0$ there.

Now if $\beta=1$ and $\alpha<1$, with $r, s, y, w$ defined as above we see that $y$ satisfies (3.3.3) on $(a, b)(-\infty<a<b<\infty)$ in case $p>0, k>q \geqslant 1$, and hence from (3.3.2) we have 


$$
\begin{aligned}
& \left(\int_{a}^{b}(x-a)^{(q-1)(\alpha(p+q)-p) / q}|u|^{p}\left|u^{\prime}\right|^{q} d x\right)^{k /(p+q)} \\
& +\left(\frac{q}{p+q}\right)^{k /(p+q)}(1-\alpha)^{1+k(q-1) /(p+q)} \\
& \times(b-a)^{(1-\alpha) k / q} \int_{a}^{b}(x-a)^{(\alpha-1) k-1}|u|^{k} d x \\
& \leqslant\left(\frac{q}{p+q}\right)^{k /(p+q)}(1-\alpha)^{1-k(p+1) /(p+q)} \\
& \quad \times(b-a)^{(1-\alpha) k / q \int_{a}^{b}(x-a)^{\alpha k-1}\left|u^{\prime}\right|^{k} d x}
\end{aligned}
$$

valid for any $u$ such that $u=\int_{a}^{x} u^{\prime} d t$ for $x \in(a, b)$, and $\int_{a}^{b}(x-a)^{\alpha k-1}\left|u^{\prime}\right|^{k} d x<\infty$ and for any $p, q, k$ such that $p>0, k>q \geqslant 1$. Equality holds in (3.4.3) if and only if $u \equiv 0$. The constant on the right side of (3.4.3) is the best possible when the left side of (3.4.3) is unchanged.

EXAMPLE 3.2. If we now set $\alpha=0$ in (3.4.3), we have

$$
\begin{aligned}
& \left(\int_{a}^{b}(x-a)^{p(1-q) / q}|u|^{p}\left|u^{\prime}\right|^{q} d x\right)^{k /(p+q)} \\
& \quad+\left(\frac{q}{p+q}\right)^{k /(p+q)}(b-a)^{k / q} \int_{a}^{b}(x-a)^{-(k+1)}|u|^{k} d x \\
& \quad \leqslant\left(\frac{q}{p+q}\right)^{k /(p+q)}(b-a)^{k / q} \int_{a}^{b}(x-a)^{-1}\left|u^{\prime}\right|^{k} d x,
\end{aligned}
$$

valid for any $u$ stated below (3.4.3) with $\alpha=0$ there.

In all previous examples we assumed that the constant $C$ in (3.2.1c) or (3.3.3c) was a finite positive number. However, we may get some examples by letting $\boldsymbol{C}=$ 0 and making certain modifications in the analysis of $\$ \S 3.2$ and 3.3. To see this we note that when $C=0$, by letting $\beta \rightarrow b-$ in (3.2.11), instead of (3.2.12) we now have

$$
\int_{a}^{b} s z^{k / a} d x \leqslant \int_{a}^{b} R\left|u^{\prime}\right|^{k} d x
$$

Now (3.2.16) is still valid for the values of $p, q, k$ set out in Theorem 3.1. Combining (3.2.16) and (3.4.5) we have

$$
\int_{a}^{b} S|u|^{k} d x \leqslant \int_{a}^{b} R\left|u^{\prime}\right|^{k} d x
$$

By the argument used in Theorem 3.1, equality can hold in (3.4.6) only if (3.2.5) 
is satisfied. By direct substitution into (3.4.6) one can verify that equality is actually attained in this case with $u$ (and $w$ ) defined by (3.2.5), provided (3.2.1c) is now replaced by the conditions:

(1 $\left.1^{\circ}\right) C=0$;

$$
\text { (2 } \left.{ }^{\circ}\right) \lim _{x \rightarrow b-} r\left(y^{\prime} / y\right)^{(k-q) / a} y^{k / q}=0 \text {. }
$$

(We also note that the second of (3.2.1c) was only needed to discuss the equality clause in Theorem 3.1.)

We immediately consider an example of (3.4.6): Suppose that $a=0, b=\infty$, $\alpha>0, \beta>0$, and $k / q>1$ with either $q \geqslant 1$ or $q<0$. Let

$$
\begin{aligned}
& r=x^{(k-q)(1-\alpha) / q}, \\
& s=\left[(\alpha+\beta) \alpha^{(k-q) / q}(k-q) / q\right] x^{\alpha+\beta-1-(\alpha k / q)}\left(1+x^{\beta}\right)^{-k / q} .
\end{aligned}
$$

Then (3.2.1) with $C=0$ has a solution

$$
y=x^{\alpha}\left(1+x^{\beta}\right)^{-\alpha / \beta} .
$$

Now by setting $w=\left(y^{\prime}\right)^{-1}$ in (3.2.3) we have, from (3.4.6),

$$
\begin{gathered}
{\left[(\alpha+\beta) \alpha^{k-1}(k-q) / q\right] \int_{0}^{\infty} x^{\alpha+\beta-\alpha k-1}\left(1+x^{\beta}\right)^{k[\alpha(q-1)-\beta] /(\beta q)}|u|^{k} d x} \\
\leqslant \int_{0}^{\infty} x^{(\alpha-1)(1-k)}\left(1+x^{\beta}\right)^{k(q-1)(\alpha+\beta) /(\beta q)}\left|u^{\prime}\right|^{k} d x
\end{gathered}
$$

valid for any $u$ such that $u=\int_{0}^{x} u^{\prime} d t$ for $x \in(0, \infty)$, and

$$
\int_{0}^{\infty} x^{(\alpha-1)(1-k)}\left(1+x^{\beta}\right)^{k(q-1)(\alpha+\beta) /(\beta q)}\left|u^{\prime}\right|^{k} d x<\infty .
$$

Equality holds in the above inequality if and only if

$$
u=k_{1} x^{\alpha}\left(1+x^{\beta}\right)^{-\alpha / \beta} \text {. }
$$

For our next example we set $k=p+q$ with $p>0, q=1$. Now, instead of (3.3.3) we consider the following differential boundary value problem:
(a) $\left(r\left(y^{\prime}\right)^{p}\right)^{\prime}+s y^{p}=0$,
(b) $\varliminf_{x \rightarrow a+} r y\left(y^{\prime}\right)^{p}<\infty$,
(c) $\left\{\begin{array}{l}\lim _{x \rightarrow b-} r\left(y^{\prime} / y\right)^{p}=c(0 \leqslant C<\infty), \\ \lim _{x \rightarrow b-} r y\left(y^{\prime}\right)^{p}<\infty,\end{array}\right.$
(d) $\left(\int_{a}^{x} r^{-1 / p} d t\right)^{p} r\left(y^{\prime} / y\right)^{p}=O(1)$ as $x \rightarrow a+$,
(e) $\int_{a}^{b} r\left(y^{\prime}\right)^{p+1} d x=\infty$. 
We note that in (3.4.7c) we allow $C=0$. In fact, the first of (3.4.7c) is no longer a restriction, since by a remark following (3.2.2), the limit concerned must exist (finite) in our case, that is the case $k=p+q$ with $p>0, q=1$. Also, the second of (3.4.7c) will coincide with the second of (3.3.3c) in the present case only when $C>0$; otherwise the second of (3.4.7c) is weaker and is all that is needed to ensure sharpness. (See also (3.3.11).) We also note that the hypothesis $y(x)=\int_{a}^{x} y^{\prime} d t$ following (3.3.3) was only needed in the proof of sharpness in order to ensure that $\int_{a}^{x} w^{-1} d t=y(x)$, when $w=\left(y^{\prime}\right)^{-1}$; but when $q=1$, this integral is not involved at all, so the restriction on $y$ can be dropped. However, the hypotheses on $r$ and $s$ are unchanged; also the hypotheses $y(x)>0, y^{\prime}(x)>0$ on $(a, b)$ are retained.

Under the boundary value problem (3.4.7) with $C=0$ there, the inequality (3.4.6) with $k=p+q(p>0, q=1)$ now becomes

$$
\int_{a}^{b} s|u|^{p+1} d x \leqslant \int_{a}^{b} r\left|u^{\prime}\right|^{p+1} d x,
$$

valid for any $u$ such that $u(x)=\int_{a}^{x} u^{\prime} d t$ for $x \in(a, b)$ and $\int_{a}^{b} r\left|u^{\prime}\right|^{p+1} d x<$ $\infty$. Equality holds in (3.4.8) if and only if $u \equiv 0$. The unit constant on the right side of (3.4.8) is the best possible. (We note that the condition $C=0$ does not affect the proof of sharpness of (3.4.8). See also Remark 3.2.)

REMARK 3.4. The inequality (3.4.8) was considered in [5], the proof being based on a class of identities due to Beesack. See also Beckenbach and Bellman [1, pp. 179, 180].

EXAMPLE 3.3. Let $\alpha>1$, and $-\infty<a<b=\infty$. Set

$$
r=(x-a)^{p+1-\alpha}, s=\left(\frac{\alpha-1}{p+1}\right)^{p+1}(x-a)^{-\alpha}, y=(x-a)^{(\alpha-1) /(p+1)} ;
$$

then we see that $y$ is a solution of (3.4.7) with $C=0$ there. From (3.4.8) with $b=\infty$, we have, for $p>0$,

$$
\int_{a}^{\infty}(x-a)^{-\alpha}|u|^{p+1} d x \leqslant\left(\frac{p+1}{\alpha-1}\right)^{p+1} \int_{a}^{\infty}(x-a)^{p+1-\alpha}\left|u^{\prime}\right|^{p+1} d x,
$$

valid for any $u$ such that $u(x)=\int_{a}^{x} u^{\prime} d t$ for $x \in(a, b)$ and $\int_{a}^{\infty}(x-a)^{p+1-\alpha}\left|u^{\prime}\right|^{p+1} d x<\infty$. Equality holds in (3.4.9) if and only if $u \equiv 0$. The constant $((p+1) /(\alpha-1))^{p+1}$ is the best possible.

EXAMPLE 3.4. Let $\alpha>1$, and $-\infty<a<b<\infty$. Let $r$, $s$ and $y$ be as in Example 3.3; then we see that $y$ is a solution of (3.4.7) with $C=$ $((\alpha-1) /(p+1))^{p}(b-a)^{1-\alpha}$ there. From (3.3.2) with $k=p+q \quad(p>0$, $q=1)$ we have, for $p>0$, 


$$
\begin{gathered}
\frac{(p+1)^{2}}{\alpha-1}(b-a)^{1-\alpha} \int_{a}^{b}|u|^{p}\left|u^{\prime}\right| d x+\int_{a}^{b}(x-a)^{-\alpha}|u|^{p+1} d x \\
\leqslant\left(\frac{p+1}{\alpha-1}\right)^{p+1} \int_{a}^{b}(x-a)^{p+1-\alpha}\left|u^{\prime}\right|^{p+1} d x,
\end{gathered}
$$

valid for any $u$ such that $u=\int_{a}^{x} u^{\prime} d t$ for every $x \in(a, b)$, and $\int_{a}^{b}(x-a)^{p+1-\alpha}\left|u^{\prime}\right|^{p+1} d x<\infty$. Equality holds in (3.4.10) if and only if $u \equiv 0$. The constant on the right side of (3.4.10) is the best possible when the left side of (3.4.10) is unchanged.

REMARK 3.5. By setting $a=0$ in (3.4.9) we get part of Hardy's inequality for integrals (see [16, Theorem 330]); the other part of Hardy's inequality for integrals may also be discussed by considering the case $u(b)=0$. (See remarks before Corollary 3.1.) More recently, a number of extensions and generalizations of Hardy's inequality have been considered by Beesack [5], Boyd [12], Tomaselli [29], and Shum [27]. (See also [8], where more references are given, for a thorough discussion of certain integral inequalities including those of Hardy's.)

REMARK 3.6. The inequality (3.4.10) is a generalization of (2.4.1) with $q=1$, and $\alpha$ replaced by $(p+1-\alpha) / p$.

\section{Chapter 4. Some Related And Reversed Inequalities}

4.1. Introduction. In Chapter 3 in order to obtain the inequality (3.2.4), our proof required that the set, $k, p, q$ satisfy either $p>0, k>q \geqslant 1$ or $p<0, k<q<0$ or $p>0, k<0<q \leqslant 1$. Since both terms on the left side of (3.2.4) are of interest to us, we shall obtain some related inequalities in which one of these terms is modified. This will also allow us to enlarge the range of $k, p, q$. To be precise, we shall consider inequalities of the following two types in $\S 4.2$ :

$$
\left(\int_{a}^{b} w^{q-1}\left|u^{\prime}\right|^{q} d x\right)^{k / q}+C^{-1} \int_{a}^{b} S|u|^{k} d x \leqslant C^{-1} \int_{a}^{b} R\left|u^{\prime}\right|^{k} d x
$$

and

$$
\left(\int_{a}^{b} W|u|^{p}\left|u^{\prime}\right|^{q} d x\right)^{k /(p+q)}+(q /(p+q))^{k /(p+q)} C^{-1} \int_{a}^{b} s z^{k / q} d x
$$

$$
\leqslant(q /(p+q))^{k /(p+q)} C^{-1} \int_{a}^{b} R\left|u^{\prime}\right|^{k} d x \quad\left(z=\int_{a}^{x} w^{q-1}\left|u^{\prime}\right|^{q} d x\right) .
$$

These inequalities will be established for appropriate values of $k, p, q$; see Theorem 4.1 and Corollaries 4.1, 4.2.

In $\S 4.3$, a reversed inequality of the inequality (3.2.4) is obtained for the 
case $p>0,0<k<q \leqslant 1$, while its sharpness (when $y$ is not admissible) is considered in $\$ 4.4$.

In the last section 4.5, a comparison of results in this and the previous chapter, with those published in other papers is given.

4.2. Some inequalities related to (3.2.4). In the following Theorem 4.1 we shall deal with some other cases of (3.2.12). We assume that $k / q>1$, or $k<0<q \leqslant 1$, since this is necessary in order to use (3.2.12). Although the following theorem and its two corollaries only deal with the case $u(a)=0$, analogous results can be proved dealing with the case $u(b)=0$.

THEOREM 4.1. Suppose that the boundary value problem (3.2.1) has a solution $y(x)$ satisfying all the hypotheses listed in Theorem 3.1. Let $w, w$, $R, S$ and $u$ be as in Theorem 3.1. Then

$$
\begin{gathered}
\left(\int_{a}^{b} w^{q-1}\left|u^{\prime}\right|^{q} d x\right)^{k / q}+C^{-1} \int_{a}^{b} S|u|^{k} d x \\
\leqslant C^{-1} \int_{a}^{b} R\left|u^{\prime}\right|^{k} d x,
\end{gathered}
$$

valid for all $p, q, k$ such that $p\langle 0, k\rangle q \geqslant 1, p+q\rangle 0$, where $C$ is defined in (3.2.1c). If, in addition, $u^{\prime}$ does not change sign on $(a, b)$, then (4.2.1) is still valid for all $p, q, k$ such that either $p>0, k<q<0, p+q<0$ or $p<0, k<0<q \leqslant 1, p+q>0$ (in the latter case the two additional assumptions- $|u(b)|<\infty$ and $\int_{a}^{b} w^{-1} d t<\infty$ - are assumed).

Also, if $p<0, k>q, 0<q \leqslant 1, p+q>0$, and $u^{\prime}$ does not change sign on $(a, b)$, then

$$
\begin{aligned}
& \left(\int_{a}^{b} W|u|^{p}\left|u^{\prime}\right|^{q} d x\right)^{k /(p+q)}+\left(\frac{q}{p+q}\right)^{k /(p+q)} C^{-1} \int_{a}^{b} s z^{k / q} d x \\
& \leqslant\left(\frac{q}{p+q}\right)^{k /(p+q)} C^{-1} \int_{a}^{b} R\left|u^{\prime}\right|^{k} d x \quad\left(z=\int_{a}^{x} w^{q-1}\left|u^{\prime}\right|^{q} d x\right)
\end{aligned}
$$

Equality holds in (4.2.1) or (4.2.2) if and only if (3.2.5) is satisfied, if $q \neq 1$, or $u=k_{1} y$, if $q=1$.

Proof. We first assume $k / q>1$; then, if $q \geqslant 1$ (and hence $k>q \geqslant$ 1), we have, from (3.2.13) with $p$ replaced by $k$,

$$
z^{k / q} \geqslant|u|^{k}\left(\int_{a}^{x} w^{-1} d t\right)^{k(1-q) / q} .
$$

Hence, 


$$
\int_{a}^{b} s z^{k / a} d x \geqslant \int_{a}^{b} S|u|^{k} d x .
$$

Combining (3.2.12) and (4.2.4) we obtain (4.2.1) with $p\langle 0, k\rangle q \geqslant 1$, $p+q>0$.

If $q<0$ (and hence $p>0, k<q<0, p+q<0$ ), then Holder's inequality gives us, if $u^{\prime}$ is of one sign,

$$
z^{1 / q} \leqslant|u|\left(\int_{a}^{x} w^{-1} d t\right)^{(1-q) / q} \text {. }
$$

Since $k<0$, from (4.2.5) we see that (4.2.3) is still valid, if $u^{\prime}$ is of one sign. Thus, repeating the argument used above we see that (4.2.1) is valid in this case.

Now, if $0<q \leqslant 1$ (and hence $k>q, 0<q \leqslant 1$ ), then (4.2.5) is still valid, if $u^{\prime}$ is also of one sign. We note that if, in addition, $p>0$, then (3.2.12) cannot be further refined. However, if $p<0$ (and hence $p<0, k\rangle q$, $0<q \leqslant 1, p+q>0$ ), and if $u^{\prime}$ is of one sign, then from (4.2.5), on noting that $k /(p+q)>0$, we have

$$
\left(\int_{a}^{b} w^{q-1}\left|u^{\prime}\right|^{q} z^{p / q} d x\right)^{k /(p+q)} \geqslant\left(\int_{a}^{b} W|u|^{p}\left|u^{\prime}\right|^{q} d x\right)^{k /(p+q)} .
$$

From (3.2.12) and the above inequality we get (4.2.2).

In this way we exhaust all possibilities concerning $k / q>1$, in which case (3.2.12) is always true. We now turn to the case $k / q<0$; in this case (3.2.12) is still valid at least for $(k<) 0<q \leqslant 1$ which is required in order to have the function $z(x)$ well defined. (See also Lemma 3.1.) Now since $0<q \leqslant 1$, hence (4.2.5) is still valid if $u^{\prime}$ is also of one sign. From (4.2.5), on noting that $k<0$, we have

$$
z^{k / q} \geqslant|u|^{k}\left(\int_{a}^{x} w^{-1} d t\right)^{k(1-q) / q} .
$$

Thus, (4.2.1) is again valid for all $k, p, q$ such that $p<0, k<0<q \leqslant 1$, $p+q>0$, if $u^{\prime}$ does not change sign.

Finally, we note that the equality conditions follow precisely as in Theorem 3.1.

Although we shall not discuss (4.2.1) further, we now consider some corollaries of (4.2.2) in Theorem 4.1 and (3.2.4) in Theorem 3.1, obtained by taking $q=1$ or $s \equiv 0$ in these theorems.

COROLlaRY 4.1. Suppose that the boundary value problem (3.2.1) has a solution $y(x)$ such that $y(x)=\int_{a}^{x} y^{\prime} d t$ with $y^{\prime}>0$ on $(a, b)$ (and $y(b)<$ $\infty$ if $k<0$ ). Finally, let $u$ be any locally absolutely continuous function on $(a, b)$, with $u(x)=\int_{a}^{x} u^{\prime} d t$, such that $\int_{a}^{b} r\left|u^{\prime}\right|^{k} d x<\infty$ (and $|u(b)|<\infty$ if 
$k<0)$. Then

$$
\begin{gathered}
\left(\int_{a}^{b}|u|^{p}\left|u^{\prime}\right| d x\right)^{k /(p+1)}+(1 /(p+1))^{k /(p+1)} C^{-1} \int_{a}^{b} s|u|^{k} d x \\
\leqslant C^{-1}\left(\frac{1}{p+1}\right)^{k /(p+1)} C^{-1} \int_{a}^{b} r\left|u^{\prime}\right|^{k} d x
\end{gathered}
$$

valid for $p>0, k>1$, where $C$ is defined in (3.2.1c). If , in addition, $u^{\prime}$ does not change sign on $(a, b)$, then (4.2.8) is still valid for all $p, k$ such that either $p>0, k<0$ or $-1<p<0, k>1$.

Equality holds in (4.2.8) if and only if $u=k_{1} y$.

Proof. By setting $q=1$ in (3.2.4) of Theorem 3.1, and (4.2.2) of Theorem 4.1 respectively, we arrive at this corollary.

COROLlaRY 4 .2. Suppose that the boundary value problem (3.2.1) with $s \equiv 0$ has a solution $y(x)$ satisfying all the hypotheses listed in Theorem 3.1. Let $w, W, R$ and $u$ be as in Theorem 3.1. Then

$$
\left(\int_{a}^{b} W|u|^{p}\left|u^{\prime}\right|^{q} d x\right)^{k /(p+q)} \leqslant\left(\frac{q}{p+q}\right)^{k /(p+q)} C^{-1} \int_{a}^{b} R\left|u^{\prime}\right|^{k} d x
$$

valid for all $p, q, k$ such that $p>0, k>q \geqslant 1$, where $C$ is defined in (3.2.1c). If, in addition, $u^{\prime}$ does not change sign on $(a, b)$, then (4.2.9) is also valid for all $p, q, k$ such that either $p<0, k<q<0$, or $p<0, k\rangle q$, $0<q \leqslant 1, p+q>0$, or $p>0, k<0<q \leqslant 1$, provided $|u(b)|<\infty$ in the latter case.

Equality holds in (4.2.9) if and only if

$$
\begin{array}{ll}
u=k_{1} y & \left(k_{1} \text { a constant }\right), \\
w=k_{2}\left(y^{\prime}\right)^{-1} & \left(k_{2}>0, a \text { constant }\right) \quad(q \neq 1),
\end{array}
$$

or $u=k_{1} y$, if $q=1$.

Proof. Setting $s \equiv 0$ in Theorem 3.1 and Theorem 4.1, then (4.2.9) follows from (3.2.4) and (4.2.2) respectively. Also, (4.2.10) is a direct consequence of (3.2.5). We note that the assumption $y(b)<\infty$ in case $k<0<\dot{q} \leqslant 1$ is retained here, so that $y$ is still admissible in this case.

REMARK 4.1. It is worth noting that (4.2.9) allows $p q<0$; this is a consequence of Theorem 4.1. Also, new examples may be obtained from (4.2.8) and (4.2.9); the latter provides generalizations of the inequalities in (2.4.2) and (2.4.3). For example, for suitable choices of $W, R$ in (4.2.9) one obtains: 


$$
\begin{aligned}
& \left(\int_{a}^{b}(x-a)^{p(1-q) / q}|u|^{p}\left|u^{\prime}\right|^{q} d x\right)^{k /(p+q)} \\
& \quad \leqslant\left(\frac{q}{p+q}\right)^{k /(p+q)}(b-a)^{(k-q) / q} \int_{a}^{b}\left|u^{\prime}\right|^{k} d x
\end{aligned}
$$

and

$$
\begin{aligned}
& \left(\int_{a}^{b}|u|^{p}\left|u^{\prime}\right|^{q} d x\right)^{k /(p+q)} \\
& \leqslant\left(\frac{q}{p+q}\right)^{1-(k p /(p+q))}(b-a)^{(k-q) /(p+q)} \\
& \cdot \int_{a}^{b}(x-a)^{p(k-1) /(p+q)}\left|u^{\prime}\right|^{k} d x .
\end{aligned}
$$

The conditions on the admissible functions $u$ and the parameters $p, q, k$ in both (4.2.11) and (4.2.12) are the same as those stated in Corollary 4.2. Equality holds in (4.2.11) if and only if $u=k_{1}(x-a)$; while equality holds in (4.2.12) if and only if $u=k_{1}(x-a)^{q /(p+q)}$. When $k=p+q,(4.2 .11)$ and (4.2.12) reduce to (2.4.2) and (2.4.3) respectively.

4.3. A reversed inequality $(p>0,0<k<q \leqslant 1)$. We are now going to deal with inequality (3.2.4) with the inequality sign reversed. We only deal with the case $u(a)=0$. The hypotheses at the beginning of $\S 3.2$ are unchanged except that instead of (3.2.1) we use the following differential boundary value problem:
(a) $\left(r\left(y^{\prime}\right)^{(k-q) / q}\right)^{\prime}+s y^{(k-q) / q}=0$,
(b) $\lim _{x \rightarrow a^{+}} r\left(y^{\prime} / y\right)^{(k-q) / q}(y(x))^{k / q}=0$,

$$
\begin{aligned}
& \text { (c) }\left\{\begin{array}{l}
\lim _{x \rightarrow b_{-}^{-}}\left(y^{\prime} / y\right)^{(k-q) / a} r(x)=C \quad(0<C<\infty), \\
\lim _{x \rightarrow b_{-}} y(x)=D<\infty,
\end{array}\right. \\
& \text { (d) } \int_{a}^{b} s y^{k / a} d x<\infty .
\end{aligned}
$$

THEOREM 4.2. Let $p>0,0<k<q \leqslant 1$, and suppose that the boundary value problem (4.3.1) has a solution $y$ such that $y(x)=\int_{a}^{x} y^{\prime} d t$ with $y^{\prime}>0$ on $(a, b)$. Let $w$ be any positive and measurable function on $(a, b)$ such that $\int_{a}^{x} w^{-1} d t<\infty$. Let $W, R$ and $S$ be defined as in Theorem 3.1. Finally, let $u$ be any locally absolutely continuous function on $(a, b)$ with $u(x)=$ $\int_{a}^{x} u^{\prime} d t$, where $u^{\prime}$ does not change sign on $(a, b)$, such that $\int_{a}^{b} S|u|^{k} d x<\infty$ 
and $\int_{a}^{b} W|u|^{p}\left|u^{\prime}\right|^{q} d x<\infty$. Then

$$
\begin{aligned}
\int_{a}^{b} R\left|u^{\prime}\right|^{k} d x \leqslant & \int_{a}^{b} S|u|^{k} d x \\
& +\left(\frac{p+q}{q}\right)^{k /(p+q)} C\left(\int_{a}^{b} W|u|^{p}\left|u^{\prime}\right|^{q} d x\right)^{k /(p+q)},
\end{aligned}
$$

where $C$ is defined in (4.3.1c).

Equality holds in (4.3.2) if and only if

$$
\begin{array}{ll}
u=k_{1} y & \left(k_{1} \text { a constant }\right), \\
w=k_{2}\left(y^{\prime}\right)^{-1} & \left(k_{2}>0, a \text { constant }\right) \quad(q \neq 1),
\end{array}
$$

or $u=k_{1} y$ if $q=1$.

Proof. For $p>0$, by using Holder's inequality, with indices $q$ and $q /(q-1)$ we have, for $0<q<1$,

$$
\begin{aligned}
|u|^{p} & =\left|\int_{a}^{x} u^{\prime} d t\right|^{p} \\
& \geqslant\left(\int_{a}^{x} w^{q-1}\left|u^{\prime}\right|^{q} d t\right)^{p / q}\left(\int_{a}^{x} w^{-1} d t\right)^{p(q-1) / q}
\end{aligned}
$$

that is,

$$
\left(\int_{a}^{x} w^{-1} d t\right)^{p(1-q) / q}|u|^{p} \geqslant\left(\int_{a}^{x} w^{q-1}\left|u^{\prime}\right|^{q} d t\right)^{p / q} .
$$

(We note that the inequality (4.3.4) is also valid for $p>0$, and $q=1$, with equality holding in this case.) Let $z(x)=\int_{a}^{x} w^{q-1}\left|u^{\prime}\right|^{q} d t$, which is well defined by (4.3.4); then from (4.3.4) we have

$$
\int_{a}^{b} W|u|^{p}\left|u^{\prime}\right|^{q} d x \geqslant \int_{a}^{b} w^{q-1}\left|u^{\prime}\right|^{q} z^{p / q} d x
$$

and, for $k / p>0$,

$$
\int_{a}^{b} S|u|^{k} d x \geqslant \int_{a}^{b} s z^{k / a} d x
$$

Now from (1.7), with $p$ replaced by $k / q$, we have, for $0<k / q<1$, and $a<\alpha<\beta<b$,

$$
\begin{gathered}
\int_{\alpha}^{\beta}\left(Q\left(v^{\prime}\right)^{k / q}+((k-q) / q)\left(G_{v}\right)^{k /(k-q)} Q^{q /(q-k)}+(k / q) G_{x}\right) d x \\
\leqslant\left.(k / q) G(v, x)\right|_{\alpha} ^{\beta} .
\end{gathered}
$$

Equality holds in (4.3.7) if and only if (3.2.8) is satisfied.

Now the proof of (4.3.2) can be completed by setting 


$$
\begin{gathered}
v=\left(\frac{q}{k}\right)\left(\frac{p+q}{q}\right)^{k /(p+q)}\left(\int_{a}^{x} w^{q-1}\left|u^{\prime}\right|^{q} z^{p / q} d t\right)^{k /(p+q)}=(q / k) z^{k / q}, \\
Q=r z^{k(q-k) / q^{2}}, \quad G=v(r h)
\end{gathered}
$$

in (4.3.7), on noting (4.3.5), (4.3.6), together with (3.2.2) and the first of (4.3.1c).

The equality clause follows from (4.3.1b,d), and the second of (4.3.1c). (We also note that (4.3.1d) and the second of (4.3.1c) assure the admissibility of $y$.)

EXAMPLE 4.1. Let $\alpha<1$, and $-\infty<a<b<\infty$, and set $r(x)=$ $(x-a)^{\alpha(k-q) / q}, s \equiv 0$. We see that (4.3.1) has a solution $y(x)=$ $(1-\alpha)^{-1}(x-a)^{1-\alpha}$ with $C=(1-\alpha)^{(k-q) / q}(b-a)^{(\alpha-1)(k-q) / q}$ in (4.3.1c). Now we set $w(x)=(x-\alpha)^{\alpha}$. All hypotheses of Theorem 4.2 are satisfied. Thus, by (4.3.2) we have

$$
\begin{aligned}
& \left(\int_{a}^{b}(x-a)^{(q-1)(\alpha(p+q)-p) / q}|u|^{p}\left|u^{\prime}\right|^{q} d x\right)^{k /(p+q)} \\
& \geqslant\left(\frac{q}{p+q}\right)^{k /(p+q)}(b-a)^{(1-\alpha)(k-q) / q} \\
& \quad \times(1-\alpha)^{1-k(1+p) /(p+q)} \int_{a}^{b}(x-a)^{\alpha(k-1)}\left|u^{\prime}\right|^{k} d x,
\end{aligned}
$$

valid for any $u$ such that $u(x)=\int_{a}^{x} u^{\prime} d t$ with $u^{\prime}$ of one sign on $(a, b)$, and

$$
\int_{a}^{b}(x-a)^{(q-1)(\alpha(p+q)-p) / q}|u|^{p}\left|u^{\prime}\right|^{q} d x<\infty,
$$

and for all $p, q, k$ such that $p>0,0<k<q \leqslant 1$. Equality holds in (4.3.10) if and only if $u=k_{1}(x-a)^{1-\alpha}$.

4.4. The sharpness of the inequality (4.3.2) when $y$ is not admissible. By setting $w=\left(y^{\prime}\right)^{-1}$ in (4.3.2), we have

$$
\begin{aligned}
& \left(\int_{a}^{b} y^{p(1-q) / q}\left(y^{\prime}\right)^{1-q}|u|^{p}\left|u^{\prime}\right|^{q} d x\right)^{k /(p+q)} \\
& \quad+(q /(p+q))^{k /(p+q)} C^{-1} \int_{a}^{b} s y^{k(1-q) / q}|u|^{k} d x \\
& \geqslant(q /(p+q))^{k /(p+q)} C^{-1} \int_{a}^{b} r\left(y^{\prime}\right)^{k(1-q) / q}\left|u^{\prime}\right|^{k} d x .
\end{aligned}
$$

We want to state the conditions under which the constant $(q /(p+q))^{k /(p+q)} C^{-1}$ is the best possible when the left side of (4.4.1) is unchanged. Now, instead of (4.3.1), we consider the following differential boundary value problem 

(a) $\left(r\left(y^{\prime}\right)^{(k-q) / q}\right)^{\prime}+s y^{(k-q) / q}=0$,
(b) $\varliminf_{x \rightarrow a+} r\left(y^{\prime} / y\right)^{(k-q) / q} y^{k / q}<\infty$,

$$
\begin{aligned}
& \text { (c) }\left\{\begin{array}{l}
\lim _{x \rightarrow b-} r\left(y^{\prime} / y\right)^{(k-q) / q}=C \quad(0<C<\infty), \\
\lim _{x \rightarrow b-} y(x)=D<\infty,
\end{array}\right. \\
& \text { (d) } \int_{a}^{b} s y^{k / q} d x=\infty
\end{aligned}
$$

We assume that (4.4.2) has a solution $y(x)=\int_{a}^{x} y^{\prime} d t$ with $y^{\prime}(x)>0$ on $(a, b)$. Note that $(4.4 .2 \mathrm{~b})$ is much weaker than the corresponding condition (4.3.1b), and that (4.4.2d) states that $y$ is not admissible. The proof of sharpness in this case is omitted, but is similar to that given following (3.3.3).

EXAMPLE 4.2. Let $\alpha>0, p>0,0<k<q=1$, and set $r(x)=$ $(x-a)^{k-1-k \alpha}, s(x)=\alpha^{k}(x-a)^{-1-k \alpha}$. Then we see that (4.4.2) has a solution $y=(x-a)^{\alpha}$ on $(a, b)(-\infty<a<b<\infty)$, with $C=\alpha^{k-1}(b-a)^{-k \alpha}$ in (4.4.2c). By (4.4.1) we have

$$
\left(\int_{a}^{b}|u|^{p}\left|u^{\prime}\right| d x\right)^{k /(p+1)}+\left(\frac{1}{p+1}\right)^{k /(p+1)} \alpha(b-a)^{k \alpha} \int_{a}^{b}(x-a)^{-1-k \alpha}|u|^{k} d x
$$

$$
\geqslant\left(\frac{1}{p+1}\right)^{k /(p+1)} \alpha^{1-k}(b-a)^{k \alpha} \int_{a}^{b}(x-a)^{k-1-k \alpha}\left|u^{\prime}\right|^{k} d x,
$$

valid for any $u$ such that $u(x)=\int_{a}^{x} u^{\prime} d t$ with $u^{\prime}$ of one sign on $(a, b)$,

$$
\int_{a}^{b}|u|^{p}\left|u^{\prime}\right| d x<\infty \text { and } \int_{a}^{b}(x-a)^{-1-k \alpha}|u|^{k} d x<\infty \text {. }
$$

Equality holds in (4.4.3) if and only if $u \equiv 0$. The constant on the right side of (4.4.3) is the best possible.

4.5. Comparison of results. The inequalities in the theorems of the previous chapter and this chapter are new. Special cases of these inequalities were considered by other authors.

In 2.5, we already compared the results in Chapter 2 with those of Boyd and Wong [11], Beesack and Das [9], and Boyd [12]. The latter paper was written from a functional analytic point of view. Since our unifying method is elementary, it may be of interest to know to what extent the result in Theorem 3.1 is comparable with that in the main theorem of [12]. The latter 
theorem of Boyd was already quoted in 2.5. It is clear that these theorems can only be compared when $s \equiv 0$ in Theorem 3.1.

We first note that when $s \equiv 0$ the inequality (3.3.2)-the sharp form of (3.2.4)-reduces to

$$
\begin{aligned}
& \left(\int_{a}^{b} y^{p(1-q) / q}\left(y^{\prime}\right)^{1-q}|u|^{p}\left|u^{\prime}\right|^{q} d x\right)^{k /(p+q)} \\
& \quad \leqslant\left(\frac{q}{p+q}\right)^{k /(p+q)} C^{-1} \int_{a}^{b} r\left(y^{\prime}\right)^{k(1-q) / q}\left|u^{\prime}\right|^{k} d x,
\end{aligned}
$$

with equality if and only if $u=k_{1} y$, where $y$ is a solution of the following boundary value problem
(a) $\left[r\left(y^{\prime}\right)^{(k-q) / q}\right]^{\prime}=0$,
(b) $\lim _{x \rightarrow a+} r\left(y^{\prime} / y\right)^{(k-q) / q} y^{k / q}=0$,
(c) $\left\{\begin{array}{l}\lim _{x \rightarrow b-} r\left(y^{\prime} / y\right)^{(k-q) / q}=C \quad(0<C<\infty), \\ \lim _{x \rightarrow b^{-}}\left[r\left(y^{\prime} / y\right)^{(k-q) / q}-C\right] y^{k / q}=0,\end{array}\right.$
(d) $r\left(y^{\prime} / y\right)^{(k-q) / q}\left(\int_{a}^{x} r^{-q /(k-q)} d t\right)^{(k-q) / q}=O(1)$ as $x \rightarrow a+$,
(e) $\int_{a}^{b} r\left(y^{\prime}\right)^{k / q} d x<\infty$.

In order to compare (4.5.1) and (4.5.2) with Boyd's main theorem in [12], we assume that $r$ is positive and measurable on $(a, b)$ with $\int_{a}^{b} r^{-q /(k-q)} d x<\infty$. (In view of the next sentence, we see that this is also a necessary and sufficient condition for (4.5.2) to have an admissible solution.) From (4.5.2a) we have $y=A \int_{a}^{x} r^{-q /(k-q)} d x$, for some constant $A$. Checking through (4.5.2b-e), we see that $y=\int_{a}^{x} r^{-q /(k-q)} d t$ is a solution of (4.5.2) with $C=\left(\int_{a}^{b} r^{-q /(k-q)} d x\right)^{(q-k) / q}$ in (4.5.2c). Now letting $p>0, k>q \geqslant 1$ (this is one of the sets of values of $k, p, q$ in Theorem 3.1), from (4.5.1) we have

$$
\begin{gathered}
\left(\int_{a}^{b}\left(\int_{a}^{x} r^{-q /(k-q)} d t\right)^{p(1-q) / q} r^{q(q-1) /(k-q)}|u|^{p}\left|u^{\prime}\right|^{q} d x\right)^{k /(p+q)} \\
\leqslant\left(\frac{q}{p+q}\right)^{k /(p+q)}\left(\int_{a}^{b} r^{-q /(k-q)} d x\right)^{(k-q) / q} \\
\times \int_{a}^{b} r^{q(k-1) /(k-q)}\left|u^{\prime}\right|^{k} d x,
\end{gathered}
$$

with equality if and only if $u=k_{1} \int_{a}^{x} r^{-q /(k-q)} d t$, for any constant $k_{1}$.

Now for the sake of convenience we write down Boyd's inequality and 
the boundary value problem $(\mathrm{P})$ as follows (see (2.5.8)):

$$
\left(\int_{a}^{b} s_{1}|u|^{p}\left|u^{\prime}\right|^{q} d x\right)^{k /(p+q)} \leqslant\left(\frac{k}{p+q}\right)^{k /(p+q)} \lambda^{k /(p+q)} \int_{a}^{b} r_{1}\left|u^{\prime}\right|^{k} d x,
$$

with equality if and only if $u=k_{1} y$, where $y$ is a solution of the boundary value problem

(1) $\left[\lambda k r_{1}\left(y^{\prime}\right)^{k-1}-q s_{1} y^{p}\left(y^{\prime}\right)^{q-1}\right]^{\prime}+p s_{1} y^{p-1}\left(y^{\prime}\right)^{q}=0$,

$$
\begin{aligned}
& \text { (2) } \lim _{x \rightarrow a+} y(x)=0, \lim _{x \rightarrow b-}\left[\lambda k r_{1}\left(y^{\prime}\right)^{k-1}-q s_{1} y^{p}\left(y^{\prime}\right)^{q-1}\right]=0, \\
& \text { (3) } \int_{a}^{b} r_{1}\left(y^{\prime}\right)^{k} d x=1
\end{aligned}
$$

We note that in [12], (4.5.4) was proved to be valid for all $p, q, k$ such that $p>0,0<q<k, k>1$.

Now if we set $r_{1}=r^{q(k-1) /(k-q)}$, and

$$
s_{1}=\left(\int_{a}^{x} r^{-q /(k-q)} d t\right)^{p(1-q) / q} r^{q(q-1) /(k-q)},
$$

then one can verify that $(\mathrm{P})$ has a solution

with

$$
y(x)=\left(\int_{a}^{b} r^{-q /(k-q)} d x\right)^{-1 / k} \int_{a}^{x} r^{-q /(k-q)} d t,
$$

$$
\lambda=(q / k)\left(\int_{a}^{b} r^{-q /(k-q)} d x\right)^{(p+q)(k-q) /(k q)} ;
$$

and with $r_{1}, s_{1}$ so defined the inequality (4.5.4) is equivalent to (4.5.3). We note, however, that our hypotheses are slightly weaker than Boyd's since he required that both $r_{1}$ and $s_{1}$ are in $C^{1}(a, b)$. On the other hand, Boyd's theorem gives (4.5.3) for the broader range of values $p>0,0<q<k, k>1$ of the parameters $p, q, k$, compared to our range $p>0,1 \leqslant q<k$.

Finally let us notice that in this special case, both boundary value problems (4.5.2) and (P) are trivial in the sense that they can be solved explicitly in terms of the function $r$. The inequality so obtained is the same inequality, that is (4.5.3), which contains Opial's inequality as a special case, as can be seen by setting $p=q=1, k=2, r \equiv 1, a=0$ in (4.5.3). Also, the inequality (4.5.3), with $q=1, k=p+1>1$, was obtained by Calvert in [14, p. 73]; the latter inequality generalized a result of Beesack in [6] who had considered the case $p=1$, as stated in $[14$, p. 73$]$.

In [14], some reversed inequalities were also given, but these inequalities 
are quite different from ours in 4.3 and 4.4. In any case, reversed inequalities of Opial type were handled in [9] systematically; these inequalities are also different from ours. They are, in fact, reversed inequalities of those in our Chapter 2, although the methods used are quite different. We have (so far) been unable to obtain reversed inequalities of those in Chapter 2 by the methods used in the last two chapters.

Another theme in the previous chapter is the generalization of Hardy's inequality for integrals in 3.4. Neither in [11] nor in [12] were the authors able to handle those cases where the solution of a certain boundary value problem fails to be admissible; and this is the case when Hardy's inequality is concerned. For an extensive bibliography related to this and similar results see [8]. In particular, our results are closely related to those in [5], [9], as well as [12]. As pointed out in 3.4, when $k=p+q, q=1$, and $C=0$ in (3.2.1c), the corresponding modified boundary value problem was handled in [5] very thoroughly. On the other hand, if we set $s \equiv 0, k=p+q$, the examples so obtained (see Remark 4.1) were essentially considered in [9]. As we noted before (in 2.5) the results in [9] were obtained without reference to any differential boundary value problem.

Acknowledgement. This is part of a thesis. The author wishes to express his sincere thanks to Professor Paul R. Beesack, Department of Mathematics, Carleton University, for his invaluable guidance and encouragement throughout this research.

\section{REFERENCES}

1. E. F. Beckenbach and R. Bellman, Inequalities, 2nd rev. ed., Ergebnisse der Mathematik and inrer Grenzgebiete, Heft 30, Springer-Verlag, New York, 1965. MR 33 \#36.

2. P. R. Beesack, Integral inequalities of the Wirtinger type, Duke Math. J. 25 (1958), 477-498. MR 20 \#3947.

3. Extensions of Wirtinger's inequality, Trans. Roy. Soc. Canada 53 (1959), 21-30.

4. - Elementary prooss of the extremal properties of the eigenvalues of the

Sturm-Liouville equation, Canad. Math. Bull. 3 (1960), 59-77. MR 23 \#A3311.

5. - Hardy's inequality and its extensions, Pacific J. Math. 11 (1961), 39-61. MR 22 \#12187.

6. - On an integral inequality of Z. Opial, Trans. Amer. Math. Soc. 104 (1962), 470-475. MR 25 \#3137.

7. - On certain discrete inequalities involving partial sums, Canad. J. Math. 21 (1969), 222-234. MR 39 \#1610.

8. - Integral inequalities involving a function and its derivative, Amer. Math. Monthly 78 (1971), 705-741.

9. P. R. Beesack and K. M., Das, Extensions of Opial's inequality, Pacific J. Math. 26 (1968), 215-232. MR 39 \#385. 
10. D. C. Benson, Inequalities involving integrals of functions and their derivatives, J. Math. Anal. Appl. 17 (1967), 292-308. MR 34 \#2809.

11. D. W. Boyd and J. S. W. Wong, An extension of Opial's inequality, J. Math. Anal. Appl. 19 (1967), 100-102. MR 35 \#3021.

12. D. W. Boyd, Best constants in a class of integral inequalities, Pacific J. Math. 30 (1969), 367-383. MR 40 \#2801.

13. - Best constants in inequalities related to Opial's inequality, J. Math. Anal. Appl. 25 (1969), 378-387. MR 38 \#4633.

14. J. Calvert, Some generalizations of Opial's inequality, Proc. Amer. Math. Soc. 18 (1967), 72-75. MR 34 \#4433.

15. K. M. Das, An inequality similar to Opial's inequality, Proc. Amer. Math. Soc. 22 (1969), 258-261. MR 39 \#5763.

16. G. H. Hardy, J. E. Littlewood and G. Pólya, Inequalities, 2nd ed., Cambridge Univ. Press, New York, 1952. MR 13, 727.

17. L.-K. Hua, On an inequality of Opial, Sci. Sinica 14 (1965), 789-790. MR 31 \#5952.

18. C.-M. Lee, On a discrete analogue of inequalities of Opial and Yang, Canad. Math. Bull. 11 (1968), 73-77. MR 39 \#387.

19. N. Levinson, On an inequality of Opial and Beesack, Proc. Amer. Math. Soc. 15 (1964), 565-566. MR 29 \#3592.

20. C. L. Mallows, An even simpler proof of Opial's inequality, Proc. Amer. Math. Soc. 16 (1965), 173. MR 30 \#1222.

21. P. M. Maroni, Sur l'inégalité d'Opial-Beesack, C. R. Acad. Sci. Paris Sér. A 264 (1967), A62-A64. MR 34 \#6007.

22. D. S. Mitrinović and P. M. Vasić, Analytic inequalities, Die Grundlehren der math. Wissenschaften, Band 165, Springer-Verlag, Berlin, 1970. MR 43 \#448.

23. C. Olech, $A$ simple proof of a certain result of $Z$. Opial, Ann. Polon. Math. 8 (1960), 61-63. MR 22 \#3773.

24. Z. Opial, Sur une inégalite', Ann. Polon. Math. 8 (1960), 29-32. MR 22 \#3772.

25. R. N. Pederson, On an inequality of Opial, Beesack and Levinson, Proc. Amer. Math. Soc. 16 (1965), 174. MR $30 \# 1223$.

26. R. Redheffer, Inequalities with three functions, J. Math. Anal. Appl. 16 (1966), 219-242. MR 35 \#321.

27. D. T. Shum, On integral inequalities related to Hardy's, Canad. Math. Bull. 14 (1971), 225-230. MR 47 \#2019.

28. - A general and sharpened form of Opial's inequality, Canad. Math. Bull. (to appear).

29. G. Tomaselli, A class of inequalities, Boll. Un. Mat. Ital. (4) 2 (1969), 622-631. MR 41 \#411.

30. J. S. W. Wong, A discrete analogue of Opial's inequality, Canad. Math. Bull. 10 (1967), 115-118. MR 35 \#3022.

31. G. -S. Yang, On a certain result of Z. Opial, Proc. Japan Acad. 42 (1966), 78-83. MR 33 \#5819.

DEPARTMENT OF MATHEMATICS, UNIVERSITY OF TORONTO, TORONTO, ONTARIO, CANADA 